DESY 06-093

hep-th/0606191

\title{
Interactions for Winding Strings in Misner Space
}

\author{
Yasuaki Hikida* \\ DESY Theory Group, Notkestrasse 85, D-22603 Hamburg, Germany
}

\begin{abstract}
We compute correlation functions of closed strings in Misner space, a big crunch big bang universe. We develop a general method for correlators with twist fields, which are relevant for the investigation on the condensation of winding tachyon. We propose to compute the correlation functions by performing an analytic continuation of the results in $\mathbb{C} / \mathbb{Z}_{N}$ Euclidean orbifold. In particular, we obtain a finite result for a general four point function of twist fields, which might be important for the interpretation as the quartic term of the tachyon potential. Three point functions are read off through the factorization, which are consistent with the known results.
\end{abstract}

*E-mail: yasuaki.hikida@desy.de 


\section{Introduction}

Lorentzian (time-dependent) orbifolds attract much attention recently, because they are solvable models describing strings in big crunch/big bang universes (see, for reviews, $[1,2,3])$. Typical Lorentzian orbifolds, however, have pathology associated with their big bang singularities. It was shown in $[4,5,6]$ that $2 \rightarrow 2$ scattering amplitude of untwisted states diverges due to graviton exchanges near the big bang singularity. Moreover, it was pointed out in [7] that a probe string induces a large backreaction due to the blue shift near the singularity, and produces a large black hole. One way to resolve the singularity is to add non-trivial directions such that the geometry has no region including the singular point $[8,5,9]$. This way of resolution, however, does not give any hint for stringy effects around the singularity.

The stringy way of resolution may come from perturbative or non-perturbative effects. A perturbative way is the condensation of winding strings, which could be massless $[10,11]$ or tachyonic $[12,13]$. It was suggested in $[10]$ that the winding strings are pair-created due to the time-dependent background, and the backreaction may resolve the singularity. On the other hand, it was conjectured in [12] that the big bang singularity is replaced by so-called tachyon state. The tachyon condensation was also investigated by D-instanton probe in $[14,15,16]$. A non-perturbative way may be given by Matrix model description of M-theory on time-dependent backgrounds. The backgrounds could be the ones with null-linear dilaton [17] as well as Lorentzian orbifolds [18, 19]. In the dual description, the singular region corresponds to the weak coupling region, where newly appearing nonAbelian fields seem to resolve the singularity. Recent developments along this direction are given, e.g., in $[20,21,22,23,24,25,26,27,28,29,30,31,32,33,34,35]$.

In this paper, we compute correlation functions of closed strings in Misner space, ${ }^{1}$ which is obtained as $(1+1)$ dimensional Minkowski space-time divided by a discrete boost. The background includes a big crunch/big bang singularity, and open and closed strings in the model have been investigated in $[39,40,10,11,41,42]$. We develop a general formalism to compute the correlation functions, in particular, we obtain the four point functions of winding strings, and find a finite result. Considering the scattering of massless winding strings, then the result suggests that the winding strings feel the singularity milder than the untwisted strings. The four point function may also give sensible information on the quartic coupling of tachyonic fields due to its finiteness.

We propose to compute the general correlation functions in the following way. First we define the Lorentzian orbifold, which describes strings in Misner space, by an analytic

\footnotetext{
${ }^{1}$ Some of the correlation functions were already computed in [11]. Though the correlators with less than two twist fields can be computed in operator formalism as in [11], it is difficult for those with more than two. It was proposed in [11] that the three point functions of twist fields can be obtained by utilizing the results of Nappi-Witten model in [36, 37, 38]. However, it is obviously important to construct a general formalism to compute the correlation functions in the Lorentzian orbifold.
} 
continuation of $\mathbb{C} / \mathbb{Z}_{N}$ Euclidean orbifold theory in the next section. In particular, we will explain in detail how to map the spectra in Misner space and in $\mathbb{C} / \mathbb{Z}_{N}$ orbifold theory. Once we have the map, then we only need to compute general correlation functions in the $\mathbb{C} / \mathbb{Z}_{N}$ theory. We start from several simple examples to get a feeling of our method in section 3 , and then we move to general ones in section 4 . We mainly follow the technique developed in $[43,44]$, where monodromy conditions around the twist fields are utilized to compute correlators in the $\mathbb{C} / \mathbb{Z}_{N}$ orbifold. Four point functions are obtained in subsection 4.1 by utilizing the method, and three point functions are deduced from the four point functions in subsections 4.2 and 4.3. In particular, the three point functions reproduce the results in [11]. In section 5, we conclude our results and discuss the physical implications of the correlation functions. In the appendix, we summarize the formula of hyergeometric function.

\section{Misner space and $\mathbb{C} / \mathbb{Z}_{N}$ orbifold}

Misner space is defined as the $(1+1)$ dimensional Minkowski space-time with the identification of the discrete boost $\left(\mathbb{R}^{1,1} / \mathbb{Z}\right)$

$$
x^{ \pm} \sim e^{ \pm 2 \pi \gamma} x^{ \pm}, \quad x^{ \pm}=\frac{1}{\sqrt{2}}\left(x_{1} \pm x_{0}\right) .
$$

Since the boost does not mix the regions inside and outside of the light-cone, the universe is divided by the lines $x^{+} x^{-}=0$. The regions with $x^{+} x^{-}<0$ are called as cosmological regions, and the regions with $x^{+} x^{-}>0$ are called as whisker regions. Changing the coordinates as $x^{ \pm}= \pm \frac{1}{\sqrt{2}} t e^{ \pm \psi}$ for $x^{+} x^{-}<0$ and $x^{ \pm}=\frac{1}{\sqrt{2}} r e^{ \pm \chi}$ for $x^{+} x^{-}>0$, the metric is given by

$$
d s^{2}=-d t^{2}+t^{2} d \psi^{2}, \quad d s^{2}=d r^{2}-r^{2} d \chi^{2},
$$

where $\psi \sim \psi+2 \pi \gamma$ and $\chi \sim \psi+2 \pi \gamma$. In the cosmological regions, the radius of space circle depends on the time, and in particular, there is a big crunch/big bang singularity at $t=0$. In the whisker regions, there are closed time-like curves due to the periodicity of the time $\chi$. These regions are conjectured to be excised from the universe by tachyon condensation [45], which will be discussed later. In the context of critical string theory, we implicitly add $d$ extra flat directions, where $d=24$ for bosonic strings and $d=8$ for superstrings.

In the following, we will connect this model with a Euclidean orbifold $\left(\mathbb{C} / \mathbb{Z}_{N}\right)$ with an enough large integer $N .^{2}$ The Euclidean orbifold is defined as 2 dimensional flat space

\footnotetext{
${ }^{2}$ In order to perform the path integral to compute correlation functions, we may have to Wick rotate the target space into a Euclidean space. In the orbifold theory, the identification by a discrete boost has to be also mapped to the one by $2 \pi / N$ rotation. It might be more transparent to use an irrational $N$ for the purpose of the Wick rotation. The assumption that $N$ is integer is only used in the next subsection to make clear the normalization of untwisted states.
} 
with the identification of $2 \pi / N$ rotation

$$
x^{ \pm} \sim e^{ \pm 2 \pi i / N} x^{ \pm}, \quad x^{ \pm}=\frac{1}{\sqrt{2}}\left(x_{1} \pm i x_{2}\right) .
$$

The condition for Misner space (2.1) may be obtained just by replacing $1 / N \leftrightarrow-i \gamma$ and $x_{2} \leftrightarrow-i x_{0}$. In the next section, we extend the orbifold procedure in the untwisted sector from the Euclidean orbifold case into the Lorentzian orbifold case. We should take a care on the fact that infinitely many images of the discrete boost should be summed over in the Lorentzian orbifold. In subsection 2.2, we propose how to define the map between the spectra of both the orbifolds. In fact, the twisted sector is found to be more subtle, and we need a trick to perform the analytic continuation.

\subsection{The untwisted sector}

We start from the states in the untwisted sector, which correspond to tachyon or graviton fields propagating the universe. Thus, the correlators involving these states provide information about the background geometry. In the 2 dimensional flat space, the tachyon field may be defined as

$$
V_{p}=e^{i \bar{p} X^{+}+i p X^{-}}, \quad\left\langle V_{p}(\infty) V_{p^{\prime}}(0)\right\rangle=(2 \pi)^{2} \delta^{(2)}\left(p-p^{\prime}\right)
$$

or in the bra and ket form as

$$
\left\langle p, \bar{p} \mid p^{\prime}, \bar{p}^{\prime}\right\rangle=(2 \pi)^{2} \delta^{(2)}\left(p-p^{\prime}\right)
$$

Here we defined $p=\frac{1}{\sqrt{2}}\left(p_{1}+i p_{2}\right)$ and $\bar{p}=\frac{1}{\sqrt{2}}\left(p_{1}-i p_{2}\right)$. In the $\mathbb{C} / \mathbb{Z}_{N}$ orbifold theory, the invariant state is given by summing over the orbifold images as

$$
|p, \bar{p}\rangle_{N}=\frac{1}{N} \sum_{n=0}^{N-1}\left|e^{\frac{2 \pi i n}{N}} p, e^{\frac{-2 \pi i n}{N}} \bar{p}\right\rangle, \quad{ }_{N}\left\langle p, \bar{p} \mid p^{\prime}, \bar{p}^{\prime}\right\rangle_{N}=(2 \pi)^{2} \frac{1}{N} \sum_{n=0}^{N-1} \delta^{(2)}\left(p-e^{\frac{2 \pi i n}{N}} p^{\prime}\right) .
$$

We can similarly define the orbifold invariant states in the Misner space as

$$
\begin{aligned}
|p, \bar{p}\rangle_{\gamma} & =\frac{1}{Z} \sum_{n \in \mathbb{Z}}\left|e^{2 \pi n \gamma} p^{+}, e^{-2 \pi n \gamma} p^{-}\right\rangle \\
\gamma\left\langle p, \bar{p} \mid p^{\prime}, \bar{p}^{\prime}\right\rangle_{\gamma} & =(2 \pi)^{2} \frac{1}{Z} \sum_{n \in \mathbb{Z}} \delta\left(p^{+}-e^{2 \pi n \gamma} p^{+^{\prime}}\right) \delta\left(p^{-}-e^{-2 \pi n \gamma} p^{-\prime}\right),
\end{aligned}
$$

where $p^{ \pm}=\frac{1}{\sqrt{2}}\left(p_{1} \pm p_{0}\right)$. Note that we divide by the infinite factor $Z=\sum_{n} 1$. The completeness conditions are

$$
1=\int \frac{d p d \bar{p}}{(2 \pi)^{2}}|p, \bar{p}\rangle\left\langle p, \bar{p}\left|=\int \frac{d p d \bar{p}}{(2 \pi)^{2}}\right| p, \bar{p}\right\rangle_{N N}\left\langle p, \bar{p}\left|=\int \frac{d p^{+} d p^{-}}{(2 \pi)^{2}}\right| p^{+}, p^{-}\right\rangle_{\gamma \gamma}\left\langle p^{+}, p^{-}\right|,
$$


which will be used to factorize four point functions into the product of two three point functions. Keeping these relations in mind, we will adopt the integral over the complex plane of $p$ and the basis for the flat space.

The standard basis in Misner space $[39,6]$ is not the one in $(2.7)$, but the both can be mapped into each other as follows. Let us define as

$$
|q, n\rangle_{N}=\frac{1}{N} \int_{0}^{N} d s\left|e^{\frac{2 \pi i s}{N}} p, e^{\frac{-2 \pi i s}{N}} \bar{p}\right\rangle e^{-2 \pi i n s},
$$

with $|p|=q$, which is also invariant under the orbifold action. The measure is given by $\sum_{n \in \mathbb{Z}} \int \frac{q d q}{(2 \pi)}$. The two point function can be computed as

$$
{ }_{N}\left\langle q, n \mid q^{\prime}, n^{\prime}\right\rangle_{N}=\frac{1}{N^{2}} \int_{0}^{N} d s \int_{0}^{N} d s^{\prime}(2 \pi)^{2} \delta^{(2)}\left(e^{\frac{2 \pi i s}{N}} q-e^{\frac{2 \pi i s^{\prime}}{N}} q^{\prime}\right) e^{2 \pi i\left(n s-n^{\prime} s^{\prime}\right)} .
$$

The phase factors are absorbed by the constant shift of $s$ and $s^{\prime}$ implicitly. Changing the variables as $\theta^{ \pm}=s \pm s^{\prime}$, we obtain

$$
\frac{1}{2 N^{2}} \int_{0}^{2 N} d \theta^{+} \int_{0}^{N} d \theta^{-}(2 \pi)^{2} \frac{1}{q} \delta\left(q-q^{\prime}\right) \delta\left(\frac{2 \pi}{N} \theta^{-}\right) e^{\pi i\left(\left(n-n^{\prime}\right) \theta^{+}+\left(n+n^{\prime}\right) \theta^{-}\right)} .
$$

The integral over $\theta^{+}$gives the delta function $2 N \delta_{n, n^{\prime}}$, and the integral over $\theta^{-}$picks up the delta function and leads $N /(2 \pi)$. Therefore, we obtain the inner product of this basis as

$$
{ }_{N}\left\langle q, n \mid q^{\prime}, n^{\prime}\right\rangle_{N}=(2 \pi) \frac{1}{q} \delta\left(q-q^{\prime}\right) \delta_{n, n^{\prime}} .
$$

The completeness condition can be also shown as

$$
\begin{aligned}
& \sum_{n} \int_{0}^{\infty} \frac{q d q}{2 \pi}|q, n\rangle_{N N}\langle q, n| \\
& =\frac{1}{N^{2}} \int_{0}^{\infty} \frac{q d q}{2 \pi} \int_{0}^{N} d s \int_{0}^{N} d s^{\prime}\left|q e^{\frac{2 \pi i s}{N}}, q e^{\left.-\frac{2 \pi i s}{N}\right\rangle\left\langle q e^{\frac{2 \pi i s^{\prime}}{N}}\right.}, q e^{-\frac{2 \pi i s^{\prime}}{N}}\right| \sum_{m} \delta\left(s-s^{\prime}+m\right) \\
& =\frac{1}{N} \int_{0}^{\infty} \frac{q d q}{(2 \pi)^{2}} \int_{0}^{2 \pi} d \psi \sum_{m}\left|q e^{i \psi}, q e^{-i \psi}\right\rangle\left\langle q e^{i \psi+\frac{2 \pi i m}{N}}, q e^{-i \psi-\frac{2 \pi i m}{N}}\right|=1 .
\end{aligned}
$$

In the first equality, the sum over $n$ is taken, which leads to the delta function. In the second equality, the integral over $s^{\prime}$ picks up the delta function, and the variable changes as $\psi=2 \pi s / N$. The last equality is the consequence of (2.8). With a simple generalization, we can define the basis in Misner space as

$$
|q, n\rangle_{\gamma}=\frac{1}{Z^{\prime}} \int_{-\infty}^{\infty} d s\left|e^{2 \pi s \gamma} p^{+}, e^{-2 \pi s \gamma} p^{-}\right\rangle e^{-2 \pi i n s}
$$

which is the same as the one in $[39,6]$. The inner product and the completeness condition are satisfied with $Z^{\prime}=\sqrt{\sum_{n} 1 / \gamma}$. In this way, we have shown that the result does not depend on the choice of the basis. 


\subsection{The twisted sectors}

As mentioned above, it is a subtle problem to define the Hilbert space of twisted sectors in Misner space. In order to clarify the problem, we first study the well-known spectrum in the $\mathbb{C} / \mathbb{Z}_{N}$ orbifold theory. In the twisted sector, the closed strings have to satisfy the twisted boundary conditions

$$
X^{ \pm}\left(e^{2 \pi i} z, e^{-2 \pi i} \bar{z}\right)=e^{ \pm \frac{2 \pi i k}{N}} X^{ \pm}(z, \bar{z})
$$

with an integer $k \neq 0 .{ }^{3}$ Therefore, the mode expansion in the $k$-th twisted sector is given by $^{4}$

$$
X^{ \pm}(z, \bar{z})=i \sum_{n} \frac{1}{\left(n \mp \frac{k}{N}\right)} \frac{\alpha_{n}^{ \pm}}{z^{n \mp \frac{k}{N}}}+i \sum_{n} \frac{1}{\left(n \pm \frac{k}{N}\right)} \frac{\tilde{\alpha}_{n}^{ \pm}}{\bar{z}^{n \pm \frac{k}{N}}}
$$

where the mode operators satisfy

$$
\left[\alpha_{m}^{-}, \alpha_{n}^{+}\right]=\left(m+\frac{k}{N}\right) \delta_{m+n}, \quad\left[\tilde{\alpha}_{m}^{-}, \tilde{\alpha}_{n}^{+}\right]=\left(m-\frac{k}{N}\right) \delta_{m+n} .
$$

The Hilbert space is generated by acting the negative mode operators to the states with quasi-zero modes (we assume $k>0$ from now on)

$$
|n, \tilde{n}, k\rangle_{N}=\left(\alpha_{0}^{+}\right)^{n}\left(\tilde{\alpha}_{0}^{-}\right)^{\tilde{n}}|k\rangle_{N}
$$

where the vacuum state has the property as

$$
\alpha_{n>0}^{+}|k\rangle_{N}=\alpha_{n \geq 0}^{-}|k\rangle_{N}=0, \quad \tilde{\alpha}_{n \geq 0}^{+}|k\rangle_{N}=\tilde{\alpha}_{n>0}^{-}|k\rangle_{N}=0 .
$$

Note that the (quasi-)zero mode part is different from one in the untwisted sector since the quasi-zero modes satisfy non-trivial commutation relations (2.17).

Naively we expect that the spectrum of twisted sectors in Misner space is given just by replacing $1 / N \leftrightarrow-i \gamma, X_{2} \leftrightarrow-i X_{0}$. Indeed, we can obtain in this way the boundary conditions

$$
X^{ \pm}\left(e^{2 \pi i} z, e^{-2 \pi i} \bar{z}\right)=e^{ \pm 2 \pi k \gamma} X^{ \pm}(z, \bar{z})
$$

and the mode expansions

$$
X^{ \pm}(z, \bar{z})=i \sum_{n} \frac{1}{(n \pm i k \gamma)} \frac{\alpha_{n}^{ \pm}}{z^{n \pm i k \gamma}}+i \sum_{n} \frac{1}{(n \mp i k \gamma)} \frac{\tilde{\alpha}_{n}^{ \pm}}{\bar{z}^{n \mp i k \gamma}}
$$

with

$$
\left[\alpha_{m}^{-}, \alpha_{n}^{+}\right]=(m-i k \gamma) \delta_{m+n}, \quad\left[\tilde{\alpha}_{m}^{-}, \tilde{\alpha}_{n}^{+}\right]=(m+i k \gamma) \delta_{m+n} .
$$

\footnotetext{
${ }^{3}$ We should assume $|k|<N$ in the $\mathbb{C} / \mathbb{Z}_{N}$ theory, but we can extend to any integer number in Misner space case.

${ }^{4}$ We set $\alpha^{\prime}=2$ throughout this paper.
} 
However, the definition of the Hilbert space is a subtle problem. As was pointed out in [39], if we define the Hilbert space as in the $\mathbb{C} / \mathbb{Z}_{N}$ orbifold, then there is no physical state. This is because the Virasoro generators are implicitly defined as

$$
\begin{aligned}
& L_{0}=-\frac{1}{2} i k \gamma(1+i k \gamma)+\alpha_{0}^{+} \alpha_{0}^{-}+\sum_{n>0}\left(\alpha_{-n}^{+} \alpha_{n}^{-}+\alpha_{-n}^{-} \alpha_{n}^{+}\right), \\
& \tilde{L}_{0}=-\frac{1}{2} i k \gamma(1+i k \gamma)+\tilde{\alpha}_{0}^{-} \tilde{\alpha}_{0}^{+}+\sum_{n>0}\left(\tilde{\alpha}_{-n}^{+} \tilde{\alpha}_{n}^{-}+\tilde{\alpha}_{-n}^{-} \tilde{\alpha}_{n}^{+}\right),
\end{aligned}
$$

and they are imaginary for every states in the Hilbert space.

A solution was proposed in [40]. In terms of Virasoro generators, the change should be made as

$$
\begin{aligned}
& L_{0}=-\frac{1}{2}(i k \gamma)^{2}+\frac{1}{2}\left(\alpha_{0}^{+} \alpha_{0}^{-}+\alpha_{0}^{-} \alpha_{0}^{+}\right)+\sum_{n>0}\left(\alpha_{-n}^{+} \alpha_{n}^{-}+\alpha_{-n}^{-} \alpha_{n}^{+}\right), \\
& \tilde{L}_{0}=-\frac{1}{2}(i k \gamma)^{2}+\frac{1}{2}\left(\tilde{\alpha}_{0}^{-} \tilde{\alpha}_{0}^{+}+\tilde{\alpha}_{0}^{+} \tilde{\alpha}_{0}^{-}\right)+\sum_{n>0}\left(\tilde{\alpha}_{-n}^{+} \tilde{\alpha}_{n}^{-}+\tilde{\alpha}_{-n}^{-} \tilde{\alpha}_{n}^{+}\right),
\end{aligned}
$$

which means that the vacuum state is not the one in (2.19) but the eigenstates as

$$
\begin{aligned}
& \frac{1}{2}\left(\alpha_{0}^{+} \alpha_{0}^{-}+\alpha_{0}^{-} \alpha_{0}^{+}\right)\left|\omega^{2}, \tilde{\omega}^{2}, k\right\rangle_{\gamma}=\omega^{2}\left|\omega^{2}, \bar{\omega}^{2}, k\right\rangle_{\gamma}, \\
& \frac{1}{2}\left(\tilde{\alpha}_{0}^{+} \tilde{\alpha}_{0}^{-}+\tilde{\alpha}_{0}^{-} \tilde{\alpha}_{0}^{+}\right)\left|\omega^{2}, \tilde{\omega}^{2}, k\right\rangle_{\gamma}=\tilde{\omega}^{2}\left|\omega^{2}, \tilde{\omega}^{2}, k\right\rangle_{\gamma} .
\end{aligned}
$$

By means of the vacuum state (2.19), this state may be expressed as

$$
\left|\omega^{2}, \tilde{\omega}^{2}, k\right\rangle_{\gamma}=\left(\epsilon_{+} \alpha_{0}^{+}\right)^{-\frac{\omega^{2}}{i k \gamma}-\frac{1}{2}}\left(\tilde{\epsilon}_{-} \tilde{\alpha}_{0}^{-}\right)^{-\frac{\tilde{\omega}^{2}}{i k \gamma}-\frac{1}{2}}|k\rangle_{\gamma},
$$

which has the extra labels $\epsilon_{+}, \tilde{\epsilon}_{-}= \pm 1$ as explained below.

Restricting to the quasi-zero mode sector, we can see the physical picture of these states. For the massive case $\omega^{2}=\tilde{\omega}^{2}=-\mathcal{M}^{2}<0$, the solutions with $\alpha_{0}^{ \pm}= \pm \epsilon \mathcal{M}$ and $\tilde{\alpha}_{0}^{ \pm}= \pm \tilde{\epsilon} \tilde{\mathcal{M}}$ lead to (now we set $z=e^{i(\tau+\sigma)}$ and $\bar{z}=e^{i(\tau-\sigma)}$ )

$$
X^{ \pm}(\tau, \sigma)= \pm \frac{2 \mathcal{M}}{k \gamma} e^{ \pm k \gamma \sigma} \sinh k \gamma \tau, \quad X^{ \pm}(\tau, \sigma)=\frac{2 \mathcal{M}}{k \gamma} e^{ \pm k \gamma \sigma} \cosh k \gamma \tau
$$

The first solution is for $\epsilon=\tilde{\epsilon}=+1$, and it describes a string winding around $\psi$-cycle and wrapping the whole cosmological regions. This type of winding string may be used to study the singularity by the winding string probe. The second solution is for $\epsilon=-\tilde{\epsilon}=+1$, and it describes a string winding around the periodic time $\chi$ and the string exists from a point $r_{0}=2 \sqrt{2} \mathcal{M} /(k \gamma)$ to a spatial infinity.

For the tachyonic case $\omega^{2}=\tilde{\omega}^{2}>0$, the solutions with $\alpha_{0}^{ \pm}=\epsilon \omega$ and $\tilde{\alpha}_{0}^{ \pm}=\tilde{\epsilon} \tilde{\omega}$ lead to

$$
X^{ \pm}(\tau, \sigma)=\frac{2 \omega}{k \gamma} e^{ \pm k \gamma \sigma} \sinh k \gamma \tau, \quad X^{ \pm}(\tau, \sigma)= \pm \frac{2 \omega}{k \gamma} e^{ \pm k \gamma \sigma} \cosh k \gamma \tau .
$$


The first solution is for $\epsilon=\tilde{\epsilon}=+1$, and it describes a string wrapping the whole whisker regions. In the context of tachyon condensation, this type of tachyon is interesting since the condensation may remove the whisker regions [45], and at the same time resolve the big bang singularity in the cosmological region [16]. The second solution is for $\epsilon=-\tilde{\epsilon}=+1$, and it describes strings pair created at a specific time $t_{0}=2 \sqrt{2} \omega /(k \gamma)$.

We need a special care on dealing with the state (2.26), since it is given by the complex power of the operators. In order to define this complex power, we utilize the Mellin transform as in [11]

$$
\left(\epsilon_{+} \alpha_{0}^{+}\right)^{-\frac{\omega^{2}}{i k \gamma}-\frac{1}{2}}=\frac{1}{\Gamma\left(\frac{\omega^{2}}{i k \gamma}+\frac{1}{2}\right)} \int_{0}^{\infty} d x x^{\frac{\omega^{2}}{i k \gamma}-\frac{1}{2}} e^{-\epsilon_{+} \alpha_{0}^{+} x}, \quad e^{-\alpha_{0}^{+} y}=\sum_{n=0}^{\infty} \frac{\left(\alpha_{0}^{+}\right)^{n}(-y)^{n}}{n !} .
$$

In other words, we define the exponential form of the state as

$$
|y, \tilde{y}, k\rangle_{N}=\sum_{n=0}^{\infty} \frac{(-y)^{n}(-\tilde{y})^{\tilde{n}}}{n ! \tilde{n} !}|n, \tilde{n}, k\rangle_{N}
$$

and perform the Wick rotation, namely, replace $X_{2} \leftrightarrow-i X_{0}$ and $1 / N \leftrightarrow-i \gamma$. Moreover, we may perform the Mellin transformation (2.29), since the above basis (2.30) is not the eigenstate of $L_{0}$ and $\tilde{L}_{0}$. In summary, it is enough to compute the correlators of the states (2.30) in the $\mathbb{C} / \mathbb{Z}_{N}$ orbifold theory since the correlators in Misner space can be obtained through the map.

\section{Simple examples}

Before moving to the full analysis of correlation functions, we start from some simple calculations. In the next subsection, we compute correlation functions with two twist fields. Since we can map two twisted fields to in and out states, we can apply operator formalism to this type of correlators. In order to compute correlators with more than two twist fields, we have to apply more general formalism, which will be developed in the next section. In subsection 3.2, we compute classical three point functions of twisted fields, which are given by the overlaps of corresponding wave functions. The both correlators were already computed in [11], but we rederive them in a bit different way such that our prescription is manifest.

\subsection{Correlation functions in operator formalism}

First we compute two point functions in the twisted sector, even though the two pint functions are nothing but the normalization of the states. For the out states, we define the bra states as

$$
{ }_{N}\langle n, \tilde{n}, k|={ }_{N}\langle k|\left(\alpha_{0}^{-}\right)^{n}\left(\tilde{\alpha}_{0}^{+}\right)^{\tilde{n}}
$$


where the vacuum state satisfies

$$
{ }_{N}\langle k| \alpha_{n \leq 0}^{+}={ }_{N}\langle k| \alpha_{n<0}^{-}=0, \quad{ }_{N}\langle k| \tilde{\alpha}_{n<0}^{+}={ }_{N}\langle k| \tilde{\alpha}_{n \leq 0}^{-}=0
$$

and

$$
{ }_{N}\left\langle k \mid k^{\prime}\right\rangle_{N}=\delta_{k, k^{\prime}}
$$

With the help of the commutation relations for the operators (2.17), we find

$$
{ }_{N}\langle n, \tilde{n}, k \mid n, \tilde{n}, k\rangle_{N}=n ! \tilde{n} !\left(\frac{k}{N}\right)^{n+\tilde{n}} \quad, \quad{ }_{N}\left\langle y, \tilde{y}, k \mid x^{\prime}, \tilde{y}^{\prime}, k\right\rangle_{N}=e^{\frac{k}{N}\left(y y^{\prime}+\tilde{y} \tilde{y}^{\prime}\right)} .
$$

In the Misner space case, $1 / N$ is replaced by $-i \gamma$. Furthermore, we have to perform the Mellin transformation (2.29), in order to change the basis into the one in (2.26). In this case, we can explicitly integrate over $y, y^{\prime}, \tilde{y}, \tilde{y}^{\prime}$, which reads

$$
\gamma\left\langle\omega^{2}, \tilde{\omega}^{2}, k \mid \omega^{\prime 2}, \tilde{\omega}^{\prime 2}, k\right\rangle_{\gamma}=\frac{\xi(-i)^{2}(i k \gamma)^{-\frac{\omega^{2}}{i k \gamma}-\frac{\tilde{\omega}^{2}}{i k \gamma}-1}}{\Gamma\left(\frac{\omega^{2}}{i k \gamma}+\frac{1}{2}\right) \Gamma\left(\frac{\tilde{\omega}^{2}}{i k \gamma}+\frac{1}{2}\right)} \delta\left(\omega^{2}-\omega^{\prime 2}\right) \delta\left(\tilde{\omega}^{2}-\tilde{\omega}^{\prime 2}\right)
$$

with $\xi=\left(\epsilon_{+} \epsilon_{-}\right)^{-\frac{\omega^{2}}{i k \gamma}-\frac{1}{2}}\left(\tilde{\epsilon}_{-} \tilde{\epsilon}_{+}\right)^{-\frac{\tilde{\omega}^{2}}{i k \gamma}-\frac{1}{2}}$. It might be convenient to change the normalization of the two point function such that the coefficient of the delta function is one.

In order to compute more hight point functions, we need to insert vertex operators. In the untwisted sector, we already have the vertex operator, which is given by the summation or the integral of (2.4) as explained in subsection 2.1. However, in the twisted sectors, it is known to be difficult to construct vertex operators. In this subsection, we therefore only compute the following three point function with the insertion of tachyon vertex operator

$$
{ }_{N}\left\langle n, \tilde{n}, k\left|: e^{i \bar{p} X^{+}+i p X^{-}}:(1)\right| n, \tilde{n}, k\right\rangle_{N},
$$

where the summation or the integration over the momentum may be needed. The three point function corresponds to the tachyon tadpole, which may represent the deformation of the geometry due to the condensation of twist field, for instance, the winding tachyon. The higher point functions can be computed if the extra vertex operators are in the untwisted sector. ${ }^{5}$

In the Hilbert space of twisted sector, we should take care of the normal ordering of the vertex operator. Here we adopt the normal ordering as

$$
: e^{i \bar{p} X^{+}+i p X^{-}}:(z)=\lim _{w \rightarrow z} e^{i \bar{p} X^{+}}(w) e^{i p X^{-}}(z) e^{-p \bar{p}\left[X_{<}^{u,+}(w), X_{>}^{u,-}(z)\right]} .
$$

The superscript $u$ denotes the mode expansion in the untwisted sector, and the subscripts $>$ and $<$ represent the positive and negative modes, respectively. In the $k$-th twisted sector, the vertex operator is explicitly written as

$$
: e^{i \bar{p} X^{+}+i p X^{-}}:=e^{i \bar{p} X_{<}^{+}+i p X_{<}^{-}} e^{i \bar{p} X_{>}^{+}+i p X_{>}^{-}} e^{i \bar{p} x_{0}^{+}+i p \tilde{x}_{0}^{-}} e^{i p x_{0}^{-}+i \bar{p} \tilde{x}_{0}^{+}} \delta_{k}^{-p \bar{p}},
$$

\footnotetext{
${ }^{5}$ See [11] for the four point function of two twist and two untwisted fields.
} 
where we have defined ( $\gamma$ is the Euler number)

$$
\begin{aligned}
& \ln \delta_{k}=\sum_{n=0}^{\infty}\left[-2 \frac{1}{n+1}+\frac{1}{n+\frac{k}{N}}+\frac{1}{n+1-\frac{k}{N}}\right]=2 \psi(1)-\psi\left(\frac{k}{N}\right)-\psi\left(1-\frac{k}{N}\right), \\
& \psi(x)=\frac{d}{d x} \ln \Gamma(x)=-\gamma-\sum_{n=0}^{\infty}\left(\frac{1}{z+n}-\frac{1}{n+1}\right),
\end{aligned}
$$

and for the quasi-zero mode parts as

$$
x_{0}^{+}=-i \frac{N}{k} \alpha_{0}^{+} z^{\frac{k}{N}}, \quad x_{0}^{-}=i \frac{N}{k} \alpha_{0}^{-} z^{-\frac{k}{N}}, \quad \tilde{x}_{0}^{+}=i \frac{N}{k} \tilde{\alpha}_{0}^{+} \bar{z}^{-\frac{k}{N}}, \quad \tilde{x}_{0}^{-}=-i \frac{N}{k} \tilde{\alpha}_{0}^{-} \bar{z}^{\frac{k}{N}} .
$$

Without the excitation of quasi-zero modes, we therefore obtain

$$
{ }_{N}\left\langle k\left|: e^{i \bar{p} X^{+}+i p X^{-}}:(1)\right| k\right\rangle_{N}=\delta_{k}^{-p \bar{p}} .
$$

This factor arises due to a higher mode effect, and the effect in Misner space was examined in [11] as stringy fuzziness.

Now we can include the excitation of quasi-zero modes. We need to compute

$$
{ }_{N}\left\langle k\left|e^{-y \alpha_{0}^{-}-\tilde{y} \tilde{\alpha}_{0}^{+}} e^{\bar{p} \frac{N}{k} \alpha_{0}^{+}+p \frac{N}{k} \tilde{\alpha}_{0}^{-}} e^{-p \frac{N}{k} \alpha_{0}^{-}-\bar{p} \frac{N}{k} \tilde{\alpha}_{0}^{+}} e^{-y^{\prime} \alpha_{0}^{+}-\tilde{y}^{\prime} \tilde{\alpha}_{0}^{-}}\right| k\right\rangle_{N}
$$

which can be evaluated by utilizing Baker-Campbell-Hausdorff formula. Gathering the quasi-zero mode and higher mode parts, we obtain

$$
{ }_{N}\left\langle y, \tilde{y}, k\left|: e^{i \bar{p} X^{+}+i p X^{-}}:(1)\right| y^{\prime}, \tilde{y}^{\prime}, k\right\rangle_{N}=e^{\frac{k}{N}\left(y y^{\prime}+\tilde{y} \tilde{y}^{\prime}\right)-\bar{p}\left(y-\tilde{y}^{\prime}\right)+p\left(y^{\prime}-\tilde{y}\right)} \delta_{k}^{-p \bar{p}} .
$$

If we insert $-i \gamma$ instead of $1 / N$, then we obtain the Misner space result, which is the same as the one in [11]. The Mellin transformation (2.29) of this function is also possible, and the result may be expressed by Tricomi's confluent hyperbolic function as

$$
\begin{array}{r}
\gamma\left\langle\omega^{2}, \tilde{\omega}^{2}, k\left|: e^{i \bar{p} X^{+}+i p X^{-}}:(1)\right| \omega^{\prime 2}, \tilde{\omega}^{\prime 2}, k\right\rangle_{\gamma}=\xi(-p)^{\frac{\omega^{2}-\omega^{\prime 2}}{i k \gamma}}(-\bar{p})^{\frac{\tilde{\omega}^{2}-\tilde{\omega}^{\prime 2}}{i k \gamma}}(i k \gamma)^{-1-\frac{\omega^{2}+\tilde{\tilde{w}}^{2}}{i k \gamma}} \\
\times U\left(\frac{\omega^{2}}{i k \gamma}+\frac{1}{2}, \frac{\omega^{2}-\omega^{\prime 2}}{i k \gamma}+1, \frac{i p \bar{p}}{k \gamma}\right) U\left(\frac{\tilde{\omega}^{2}}{i k \gamma}+\frac{1}{2}, \frac{\tilde{\omega}^{2}-\tilde{\omega}^{\prime 2}}{i k \gamma}+1, \frac{i p \bar{p}}{k \gamma}\right) \delta_{k}^{-p \bar{p}}
\end{array}
$$

where the integral representation

$$
U(a, b, z)=\frac{1}{\Gamma(a)} \int_{0}^{\infty} e^{-z t} t^{a-1}(t+1)^{-a+b-1} d t
$$

is used. As discussed before, the condensation of winding tachyon may deform the geometry near the big bang singularity in the cosmological region. This result suggests that a stringy effect enhances the deformed region due to the string fuzziness. 


\subsection{Classical three point functions of twist fields}

The wave functions corresponding to the twisted states in $\mathbb{C} / \mathbb{Z}_{N}$ theory can be constructed as follows. Because the quasi-zero modes satisfy the commutation relations (2.17), the operators may be represented as

$$
\alpha_{0}^{ \pm}=-i \partial_{\mp} \pm i \frac{k}{2 N} x^{ \pm}, \quad \quad \tilde{\alpha}_{0}^{ \pm}=-i \partial_{\mp} \mp i \frac{k}{2 N} x^{ \pm} .
$$

The definition of the vacuum state (2.19) gives the condition that the corresponding wave function $\Psi_{k}^{+}$should be annihilated by $\alpha_{0}^{-}$and $\tilde{\alpha}_{0}^{+}$. The condition leads that the wave function is of the Gaussian form as

$$
\Psi_{k}^{+}\left(x^{ \pm}\right)=C e^{-\frac{k}{2 N} x^{+} x^{-}}, \quad \Psi_{k}^{-}\left(x^{ \pm}\right)\left(=\Psi_{k}^{+}\left(x^{ \pm}\right)^{*}\right)=C^{*} e^{-\frac{k}{2 N} x^{+} x^{-}} .
$$

The normalization is fixed as $C=\sqrt{\frac{k}{N \pi}}$, so that $\int d x^{2} \Psi_{k}^{-} \Psi_{k}^{+}=1$. The three point function without excitation is then computed as the overlap of the wave functions as $\left(k_{1}=k_{2}+k_{3}\right)$

$$
C_{3}=\int d x^{2} \Psi_{k_{1}}^{\mp} \Psi_{k_{2}}^{ \pm} \Psi_{k_{3}}^{ \pm}=\frac{1}{\sqrt{\pi}} \sqrt{\frac{\frac{k_{2}}{N} \frac{k_{3}}{N}}{\frac{k_{1}}{N}}} .
$$

The inclusion of excitation is given by acting the creation operators to the vacuum wave function as

$$
\Psi_{k}^{+, n, \tilde{n}}=\left(\alpha_{0}^{+}\right)^{n}\left(\tilde{\alpha}_{0}^{-}\right)^{\tilde{n}} \Psi_{k}^{+}=\left(-i \partial_{-}+i \frac{k}{2 N} x^{+}\right)^{n}\left(-i \partial_{+}+i \frac{k}{2 N} x^{-}\right)^{\bar{n}} \Psi_{k}^{+}
$$

and also $\Psi_{k}^{-, n, \tilde{n}}=\left(\Psi_{k}^{+, \tilde{n}, n}\right)^{*}$.

Using the above wave functions, now we can compute the overlaps of the excited wave functions, for example, as

$$
\begin{gathered}
\int d x^{2} \Psi_{k_{1}}^{\mp, m, 0} \Psi_{k_{2}}^{ \pm, n, 0} \Psi_{k_{3}}^{ \pm}=\delta_{m, n} m !\left(\frac{k_{2}}{N}\right)^{m} C_{3} \\
\int d x^{2} \Psi_{k_{1}}^{\mp} \Psi_{k_{2}}^{ \pm, n, 0} \Psi_{k_{3}}^{ \pm, 0, \tilde{n}}=\delta_{n, \tilde{n}} n !\left(-\frac{\frac{k_{2}}{N} \frac{k_{3}}{N}}{\frac{k_{1}}{N}}\right)^{n} C_{3}
\end{gathered}
$$

Non-trivial overlaps may be

$$
\int d x^{2} \Psi_{k_{1}}^{\mp} \Psi_{k_{2}}^{ \pm, n, \tilde{n}} \Psi_{k_{3}}^{ \pm}=\delta_{n, \tilde{n}} n !\left(\frac{\frac{k_{2}}{N} \frac{k_{3}}{N}}{\frac{k_{1}}{N}}\right)^{n} C_{3}
$$

and

$$
\int d x^{2} \Psi_{k_{1}}^{\mp, n \tilde{n}} \Psi_{k_{2}}^{ \pm} \Psi_{k_{3}}^{ \pm}=0
$$

where we should use

$$
\alpha_{0}^{+} \tilde{\alpha}_{0}^{-}=-\frac{k}{N} \frac{k}{N} x^{+} x^{-}+\frac{k}{N} .
$$


In the language of the basis

$$
\Psi_{k}^{ \pm, y, \tilde{y}}=\sum_{n, \tilde{n}} \frac{(-y)^{n}(-\tilde{y})^{\tilde{n}}}{n ! \tilde{n} !} \Psi_{k}^{ \pm, n, \tilde{n}}
$$

the three point functions can be summarized as

$$
\int d x^{2} \Psi_{k_{1}}^{\mp, y_{1}, \tilde{y}_{1}} \Psi_{k_{2}}^{ \pm, y_{2}, \tilde{y}_{2}} \Psi_{k_{3}}^{ \pm, y_{3}, \tilde{y}_{3}}=e^{y_{3}\left(\frac{k_{1}}{N} y_{1}+\frac{k_{2}}{N} y_{2}\right)+\tilde{y}_{3}\left(\frac{k_{1}}{N} \tilde{y}_{1}+\frac{k_{2}}{N} \tilde{y}_{2}\right)+\frac{\frac{k_{2}}{N} \frac{k_{3}}{N}}{\frac{k_{1}}{N}}\left(y_{2}-y_{3}\right)\left(\tilde{y}_{2}-\tilde{y}_{3}\right)} C_{3} .
$$

Replacing $1 / N$ by $-i \gamma$, we reproduce the result in [11]. In subsection 4.2 , we derive the correlators in the full quantum level by utilizing the monodromy conditions.

\section{Correlation functions}

In the $\mathbb{C} / \mathbb{Z}_{N}$ orbifold model, the coordinate fields $X^{ \pm}$have twisted boundary conditions (2.15), and the twist can be generated by the insertion of twist field $\sigma_{k}^{ \pm}(k>0)$, whose operator product expansions (OPEs) are

$$
\begin{aligned}
& i \partial X^{+}(z) \sigma_{k}^{+}(w, \bar{w}) \sim \frac{\tau^{+}(w, \bar{w})}{(z-w)^{1-\frac{k}{N}}}, i \partial X^{-}(z) \sigma_{k}^{+}(w, \bar{w}) \sim \frac{\tau^{+\prime}(w, \bar{w})}{(z-w)^{\frac{k}{N}}}, \\
& i \bar{\partial} X^{+}(\bar{z}) \sigma_{k}^{+}(w, \bar{w}) \sim \frac{\tilde{\tau}^{+\prime}(w, \bar{w})}{(\bar{z}-\bar{w})^{\frac{k}{N}}}, \quad i \bar{\partial} X^{-}(\bar{z}) \sigma_{k}^{+}(w, \bar{w}) \sim \frac{\tilde{\tau}^{+}(w, \bar{w})}{(\bar{z}-\bar{w})^{1-\frac{k}{N}}},
\end{aligned}
$$

and similar ones for $\sigma_{k}^{-}$. These twist fields are related to the vacuum states in the twisted sector by

$$
\lim _{z \rightarrow 0} \sigma_{k}^{+}(z)|0\rangle_{N}=|k\rangle_{N}, \quad \quad \quad \lim _{z \rightarrow \infty}\left(-z^{2}\right)^{h_{k}}{ }_{N}\langle 0| \sigma_{k}^{-}(z)={ }_{N}\langle k|
$$

where we denote the conformal weight as $h_{k}=\frac{1}{2} \frac{k}{N}\left(1-\frac{k}{N}\right)$. We can define the twist fields with the excitation of quasi-zero modes by repeating the OPEs (4.1) as

$$
\begin{gathered}
:\left(i \partial X^{+}\right)^{n}\left(i \bar{\partial} X^{-}\right)^{\tilde{n}}:(z, \bar{z}) \sigma_{k}^{+}(w, \bar{w}) \sim \frac{\sigma_{k}^{+, n, \bar{n}}(w, \bar{w})}{(z-w)^{n\left(1-\frac{k}{N}\right)}(\bar{z}-\bar{w})^{\tilde{n}\left(1-\frac{k}{N}\right)}}, \\
\sigma_{k}^{-}(z, \bar{z}):\left(i \partial X^{-}\right)^{n}\left(i \bar{\partial} X^{+}\right)^{\tilde{n}}:(w, \bar{w}) \sim \frac{\sigma_{k}^{-, n, \bar{n}}(w, \bar{w})}{(z-w)^{n\left(1-\frac{k}{N}\right)}(\bar{z}-\bar{w})^{\tilde{n}\left(1-\frac{k}{N}\right)}} .
\end{gathered}
$$

The orderings are chosen to be consistent with the definitions (4.2) and (2.18), (3.2). This definition of excited twist fields implies $\sigma_{k}^{+, 1,0}=\tau^{+}, \sigma_{k}^{+, 0,1}=\tilde{\tau}^{+}$. Note that $\tau^{+\prime}$ and $\tilde{\tau}^{+\prime}$ include not quasi-zero modes but higher excited modes. We can also define

$$
\sigma_{k}^{ \pm, y, \tilde{y}}=\sum_{n, \tilde{n}=0}^{\infty} \frac{(-y)^{n}(-\tilde{y})^{\tilde{n}}}{n ! \tilde{n} !} \sigma_{k}^{ \pm, n, \tilde{n}}
$$


and $\sigma_{k}^{ \pm, \omega^{2}, \tilde{\omega}^{2}}$ by replacing $1 / N \leftrightarrow-i \gamma, X_{2} \leftrightarrow-i X_{0}$ and performing the Mellin transformation (2.29). In the following, we will try to compute correlation functions involving the twist fields of the form (4.4).

We will utilize the monodromy conditions around twist fields as in [43]. This method may be applied to any correlators, but we mainly compute four point functions and read off the three point functions through the factorization. We begin with the four point function without excitation

$$
Z_{N}\left(z_{i}, \bar{z}_{i}\right)=\left\langle\prod_{i=1}^{4} \sigma_{k_{i}}^{\epsilon_{i}}\left(z_{i}, \bar{z}_{i}\right)\right\rangle_{N}
$$

where $k_{1}+k_{3}=k_{2}+k_{4}$ and $\epsilon_{1}=-\epsilon_{2}=\epsilon_{3}=-\epsilon_{4}=-1$. The green functions in the presence of the twist fields are defined as

$$
g\left(z, w ; z_{i}, \bar{z}_{i}\right)=\frac{\left\langle-\partial X^{+}(z) \partial X^{-}(w) \prod_{i=1}^{4} \sigma_{k_{i}}^{\epsilon_{i}}\left(z_{i}, \bar{z}_{i}\right)\right\rangle_{N}}{\left\langle\prod_{i=1}^{4} \sigma_{k_{i}}^{\epsilon_{i}}\left(z_{i}, \bar{z}_{i}\right)\right\rangle_{N}},
$$

and

$$
h\left(\bar{z}, w ; z_{i}, \bar{z}_{i}\right)=\frac{\left\langle-\bar{\partial} X^{+}(\bar{z}) \partial X^{-}(w) \prod_{i=1}^{4} \sigma_{k_{i}}^{\epsilon_{i}}\left(z_{i}, \bar{z}_{i}\right)\right\rangle_{N}}{\left\langle\prod_{i=1}^{4} \sigma_{k_{i}}^{\epsilon_{i}}\left(z_{i}, \bar{z}_{i}\right)\right\rangle_{N}} .
$$

We also define the other green functions $\bar{g}(\bar{z}, \bar{w})$ and $\bar{h}(z, \bar{w})$ by interchanging $\partial \leftrightarrow \bar{\partial}$.

The excitation of quasi-zero modes can be incorporated by utilizing the green functions as

$$
\begin{aligned}
& \left\langle\sigma_{k_{1}}^{-, n, 0}\left(z_{1}\right) \sigma_{k_{2}}^{+, n, 0}\left(z_{2}\right) \sigma_{k_{3}}^{-}\left(z_{3}\right) \sigma_{k_{4}}^{+}\left(z_{4}\right)\right\rangle_{N} \\
& =\lim _{z \rightarrow z_{2}, w \rightarrow z_{1}}\left(z-z_{2}\right)^{n\left(1-\frac{k_{2}}{N}\right)}\left(z_{1}-w\right)^{n\left(1-\frac{k_{1}}{N}\right)}\left\langle\left(i \partial X^{+}(z)\right)^{n}\left(i \partial X^{-}(w)\right)^{n} \prod_{i=1}^{4} \sigma_{k_{i}}^{\epsilon_{i}}\left(z_{i}, \bar{z}_{i}\right)\right\rangle_{N} \\
& =n ! g^{\prime}\left(z_{2}, z_{1} ; z_{i}, \bar{z}_{i}\right)^{n}\left\langle\prod_{i=1}^{4} \sigma_{k_{i}}^{\epsilon_{i}}\left(z_{i}, \bar{z}_{i}\right)\right\rangle_{N},
\end{aligned}
$$

where we have defined

$$
g^{\prime}\left(z_{2}, z_{1} ; z_{i}, \bar{z}_{i}\right)=\lim _{z \rightarrow z_{2}, w \rightarrow z_{1}}\left(z-z_{2}\right)^{1-\frac{k_{2}}{N}}\left(z_{1}-w\right)^{1-\frac{k_{1}}{N}} g\left(z, w ; z_{i} \cdot \bar{z}_{i}\right) .
$$

Recall that we have defined the excited states through the OPEs (4.3). We will also use the functions $h^{\prime}, \bar{g}^{\prime}, \bar{h}^{\prime}$, which are defined in the same procedure to take the limits. It is straightforward to convert into the basis (4.4) as

$$
\left\langle\sigma_{k_{1}}^{-, y_{1}, 0}\left(z_{1}\right) \sigma_{k_{2}}^{+, y_{2}, 0}\left(z_{2}\right) \sigma_{k_{3}}^{-}\left(z_{3}\right) \sigma_{k_{4}}^{+}\left(z_{4}\right)\right\rangle_{N}=e^{y_{2} y_{1} g^{\prime}\left(z_{2}, z_{1} ; z_{i}, \bar{z}_{i}\right)}\left\langle\prod_{i=1}^{4} \sigma_{k_{i}}^{\epsilon_{i}}\left(z_{i}, \bar{z}_{i}\right)\right\rangle_{N} .
$$


Generalizing this, we can write down the four point functions with general excitations in terms of $g^{\prime}, \bar{g}^{\prime}, h^{\prime}, \bar{h}^{\prime}$ as

$$
\begin{aligned}
\left\langle\prod_{i=1}^{4} \sigma_{k_{i}}^{\epsilon_{i}, y_{i}, \tilde{y}_{i}}\left(z_{i}\right)\right\rangle_{N} & =e^{y_{2} y_{1} g^{\prime}\left(z_{2}, z_{1}\right)+y_{2} y_{3} g^{\prime}\left(z_{2}, z_{3}\right)+y_{4} y_{1} g^{\prime}\left(z_{4}, z_{1}\right)+y_{4} y_{3} g^{\prime}\left(z_{4}, z_{3}\right)} \\
& \times e^{\tilde{y}_{1} y_{1} h^{\prime}\left(\bar{z}_{1}, z_{1}\right)+\tilde{y}_{1} y_{3} h^{\prime}\left(\bar{z}_{1}, z_{3}\right)+\tilde{y}_{3} y_{1} h^{\prime}\left(\bar{z}_{3}, z_{1}\right)+\tilde{y}_{3} y_{3} h^{\prime}\left(\bar{z}_{3}, z_{3}\right)} \\
& \times e^{\tilde{y}_{1} \tilde{y}_{2} \bar{g}^{\prime}\left(\bar{z}_{1}, \bar{z}_{2}\right)+\tilde{y}_{1} \tilde{y}_{4} \bar{g}^{\prime}\left(\bar{z}_{1}, \bar{z}_{4}\right)+\tilde{y}_{3} \tilde{y}_{2} \bar{g}^{\prime}\left(\bar{z}_{3}, \bar{z}_{2}\right)+\tilde{y}_{3} \tilde{y}_{4} \bar{g}^{\prime}\left(\bar{z}_{3}, \bar{z}_{4}\right)} \\
& \times e^{y_{2} \tilde{y}_{2} \bar{h}^{\prime}\left(z_{2}, \bar{z}_{2}\right)+y_{4} \tilde{y}_{2} \bar{h}^{\prime}\left(z_{4}, \bar{z}_{2}\right)+y_{2} \tilde{y}_{4} \bar{h}^{\prime}\left(z_{2}, \bar{z}_{4}\right)+y_{4} \tilde{y}_{4} \bar{h}^{\prime}\left(z_{4}, \bar{z}_{4}\right)}\left\langle\prod_{i=1}^{4} \sigma_{k_{i}}^{\epsilon_{i}}\left(z_{i}, \bar{z}_{i}\right)\right\rangle_{N}
\end{aligned}
$$

The explicit form will be determined through the monodromy conditions below.

The three point functions can be read off from the above four point function (4.11) though the factorization as follows. Consider the four point function of primary fields

$$
\left\langle\prod_{i=1}^{4} \Phi_{i}\left(z_{i}, \bar{z}_{i}\right)\right\rangle
$$

We suppose that the primary fields $\Phi_{i}$ have the conformal weights $\left(h_{i}, \bar{h}_{i}\right)$. If we take the limit of $z_{1} \rightarrow z_{2}$ and $z_{3} \rightarrow z_{4}$, then it is useful to use the OPEs of the primary fields

$$
\Phi_{1}\left(z_{1}, \bar{z}_{1}\right) \Phi_{2}\left(z_{2}, \bar{z}_{2}\right)=\sum_{I} \frac{C_{12}^{I} \Phi_{I}\left(z_{2}, \bar{z}_{2}\right)}{z_{12}^{\Delta_{12}} \bar{z}_{12}^{\bar{\Delta}_{12}}}, \quad \Phi_{3}\left(z_{3}, \bar{z}_{3}\right) \Phi_{4}\left(z_{4}, \bar{z}_{4}\right)=\sum_{I} \frac{C_{34}^{I} \Phi_{I}\left(z_{4}, \bar{z}_{4}\right)}{z_{34}^{\Delta_{34}} \bar{z}_{34}^{\bar{\Delta}_{34}}}
$$

where $z_{12}=z_{1}-z_{2}, \Delta_{12}=h_{1}+h_{2}-h_{I}$, and so on. Therefore, in this limit, the leading term of the four point function is given by the product of three point functions as

$$
\left\langle\prod_{i=1}^{4} \Phi_{i}\left(z_{i}, \bar{z}_{i}\right)\right\rangle \sim \sum_{p} \frac{1}{z_{24}^{2 h_{p}} \bar{z}_{24}^{2 \bar{h}_{I}}} \frac{C_{12}^{I}}{z_{12}^{\Delta_{12}} \bar{z}_{12}^{\bar{\Delta}_{12}}} \frac{C_{34 I}}{z_{34}^{\Delta_{34}} \bar{z}_{34}^{\bar{\Delta}_{34}}} .
$$

In the following analysis, we may set $\left(z_{1}, z_{2}, z_{3}, z_{4}\right)=(0, x, 1, \infty)$, and take the limit of $x \rightarrow 0$ or $x \rightarrow \infty$. When we take the limit of $x \rightarrow 0$, we use the OPE between $\Phi_{2}$ and $\Phi_{1}$, and when we take the limit of $x \rightarrow \infty$, we use the OPE between $\Phi_{2}$ and $\Phi_{4}$.

In the next subsection, we compute a general four point function by following [43]. After that, we deduce the three point functions of twisted fields in subsection 4.2, which are the quantum version of the ones in subsection 3.2. Then, we move to the three point functions with an untwisted fields in subsection 4.3, which is found to be consistent with the results in subsection 3.1.

\subsection{Four point functions}

We start from the four point function of twist fields without excitation (4.5), which will be determined from the green functions (4.6) and (4.7) as explained below. Utilizing 
the monodromy conditions (4.1), we can fix the form of the green function (4.6) almost uniquely as ${ }^{6}$

$$
g\left(z, w ; z_{i}, \bar{z}_{i}\right)=\omega_{k}(z) \omega_{N-k}(w)\left[\frac{P\left(z, w, z_{i}\right)}{(z-w)^{2}}+A\left(z_{i}, \bar{z}_{i}\right)\right]
$$

with unfixed function $A\left(z_{i}, \bar{z}_{i}\right)$. The classical parts $\partial X^{+}(z)=\omega_{k}(z)$ and $\partial X^{-}(z)=$ $\omega_{N-k}(z)$ are determined as

$$
\begin{gathered}
\omega_{k}(z)=\left(z-z_{1}\right)^{-\frac{k_{1}}{N}}\left(z-z_{2}\right)^{-1+\frac{k_{2}}{N}}\left(z-z_{3}\right)^{-\frac{k_{3}}{N}}\left(z-z_{4}\right)^{-1+\frac{k_{4}}{N}}, \\
\omega_{N-k}(z)=\left(z-z_{1}\right)^{-1+\frac{k_{1}}{N}}\left(z-z_{2}\right)^{-\frac{k_{2}}{N}}\left(z-z_{3}\right)^{-1+\frac{k_{3}}{N}}\left(z-z_{4}\right)^{-\frac{k_{4}}{N}},
\end{gathered}
$$

which reproduces the monodromy (4.1). The function $P\left(z, w, z_{i}\right)$ is fixed to reproduce the singular behavior $i \partial X^{+}(z) i \partial X^{-}(w) \sim 1 /(z-w)^{2}$ as

$$
\begin{aligned}
& P\left(z, w, z_{i}\right)=\left[\frac{k_{1}}{N}-\frac{k_{2}}{N}\right]\left(z-z_{1}\right)\left(z-z_{2}\right)\left(w-z_{3}\right)\left(w-z_{4}\right) \\
& \quad+\frac{k_{2}}{N}\left(z-z_{1}\right)\left(z-z_{3}\right)\left(w-z_{2}\right)\left(w-z_{4}\right)+\left[1-\frac{k_{4}}{N}\right]\left(z-z_{2}\right)\left(z-z_{4}\right)\left(w-z_{1}\right)\left(w-z_{3}\right) \\
& \quad+\left[\frac{k_{3}}{N}-\frac{k_{2}}{N}\right]\left(z-z_{2}\right)\left(z-z_{3}\right)\left(w-z_{1}\right)\left(w-z_{4}\right) .
\end{aligned}
$$

In particular, there should be no single pole for $z \sim w$. This function is unique up to the shift of $A\left(z_{i}, \bar{z}_{i}\right)$.

The function $A\left(z_{i}, \bar{z}_{i}\right)$ can be determined by global monodromy conditions

$$
\oint_{\mathcal{C}_{i}} d z \partial X^{+}+\oint_{\mathcal{C}_{i}} d \bar{z} \bar{\partial} X^{+}=0
$$

where the contours $\mathcal{C}_{i}(i=1,2)$ are taken so that $X^{ \pm}$receive totally no phase factors around the twist fields inside the contours. Here we take the closed loops $\mathcal{C}_{1}$ rounding $k_{2}$ times around $z_{1}$ and $k_{1}$ times around $z_{2}$ and $\mathcal{C}_{2}$ rounding $k_{3}$ times around $z_{2}$ and $k_{2}$ times around $z_{3}$. The conditions (4.18) may be expressed as

$$
\oint_{\mathcal{C}_{i}} d z g(z, w)+\oint_{\mathcal{C}_{i}} d \bar{z} h(\bar{z}, w)=0
$$

where $h(\bar{z}, w)$ is defined in (4.7). This green function is also determined up to unknown function $B\left(z_{i}, \bar{z}_{i}\right)$ as

$$
h\left(\bar{z}, w ; z_{i}, \bar{z}_{i}\right)=\bar{\omega}_{N-k}(\bar{z}) \omega_{N-k}(w) B\left(z_{i}, \bar{z}_{i}\right) .
$$

Because we have now two independent global monodromy conditions (4.19), we can uniquely fix the undetermined functions $A\left(z_{i}, \bar{z}_{i}\right)$ and $B\left(z_{i}, \bar{z}_{i}\right)$.

\footnotetext{
${ }^{6}$ We should note that the assumption of integer $N$ is not needed here and below. In fact, the four point function (4.5) was already calculated for an irrational orbifold case in [36] as a free field realization of Nappi-Witten model.
} 
Let us solve the conditions (4.19). We first take $w \rightarrow \infty$, and then set $z_{1}=0, z_{2}=x$, $z_{3}=1$ and $z_{4} \rightarrow \infty$. Using the integral representation of hypergeometric function (A.2), the conditions (4.19) now read

$$
\begin{aligned}
& \hat{A} x^{\frac{k_{2}}{N}-\frac{k_{1}}{N}} \frac{\Gamma\left(1-\frac{k_{1}}{N}\right) \Gamma\left(\frac{k_{2}}{N}\right)}{\Gamma\left(1-\frac{k_{1}}{N}+\frac{k_{2}}{N}\right)} F_{1}(x)+\hat{B} \bar{x}^{\frac{k_{1}}{N}-\frac{k_{2}}{N}} \frac{\Gamma\left(\frac{k_{1}}{N}\right) \Gamma\left(1-\frac{k_{2}}{N}\right)}{\Gamma\left(1-\frac{k_{2}}{N}+\frac{k_{1}}{N}\right)} \bar{G}_{1}(\bar{x}) \\
& =\left(1-\frac{k_{4}}{N}\right) x^{1-\frac{k_{1}}{N}+\frac{k_{2}}{N}} \frac{\Gamma\left(1-\frac{k_{1}}{N}\right) \Gamma\left(1+\frac{k_{2}}{N}\right)}{\Gamma\left(2-\frac{k_{1}}{N}+\frac{k_{2}}{N}\right)} K_{1}(x), \\
& \hat{A}(1-x)^{\frac{k_{2}}{N}-\frac{k_{3}}{N}} \frac{\Gamma\left(1-\frac{k_{3}}{N}\right) \Gamma\left(\frac{k_{2}}{N}\right)}{\Gamma\left(1-\frac{k_{3}}{N}+\frac{k_{2}}{N}\right)} F_{2}(1-x)-\hat{B}(1-\bar{x})^{\frac{k_{3}}{N}-\frac{k_{2}}{N}} \frac{\Gamma\left(\frac{k_{3}}{N}\right) \Gamma\left(1-\frac{k_{2}}{N}\right)}{\Gamma\left(1-\frac{k_{2}}{N}+\frac{k_{3}}{N}\right)} \bar{G}_{2}(1-\bar{x}) \\
& =-\left(1-\frac{k_{4}}{N}\right)(1-x)^{1-\frac{k_{3}}{N}+\frac{k_{2}}{N}} \frac{\Gamma\left(1-\frac{k_{3}}{N}\right) \Gamma\left(1+\frac{k_{2}}{N}\right)}{\Gamma\left(2-\frac{k_{3}}{N}+\frac{f_{2}}{N}\right)} K_{2}(1-x),
\end{aligned}
$$

where

$$
\begin{aligned}
& F_{1}(x)=F\left(\frac{k_{3}}{N}, 1-\frac{k_{1}}{N}, 1-\frac{k_{1}}{N}+\frac{k_{2}}{N} ; x\right), F_{2}(1-x)=F\left(\frac{k_{1}}{N}, 1-\frac{k_{3}}{N}, 1-\frac{k_{3}}{N}+\frac{k_{2}}{N} ; 1-x\right), \\
& \bar{G}_{1}(\bar{x})=F\left(1-\frac{k_{3}}{N}, \frac{k_{1}}{N}, 1+\frac{k_{1}}{N}-\frac{k_{2}}{N} ; \bar{x}\right), \bar{G}_{2}(1-\bar{x})=F\left(1-\frac{k_{1}}{N}, \frac{k_{3}}{N}, 1-\frac{k_{2}}{N}+\frac{k_{3}}{N} ; 1-\bar{x}\right), \\
& K_{1}(x)=F\left(\frac{k_{3}}{N}, 1-\frac{k_{1}}{N}, 2-\frac{k_{1}}{N}+\frac{k_{2}}{N} ; x\right), K_{2}(1-x)=F\left(\frac{k_{1}}{N}, 1-\frac{k_{3}}{N}, 2-\frac{k_{3}}{N}+\frac{k_{2}}{N} ; 1-x\right) .
\end{aligned}
$$

Here we have defined

$$
\hat{A}(x, \bar{x})=-\lim _{z_{4} \rightarrow \infty} z_{4} A\left(0, x, 1, z_{4}\right), \quad \hat{B}(x, \bar{x})=\lim _{z_{4} \rightarrow \infty}\left|z_{4}\right|^{-\frac{2 k_{4}}{N}} B\left(0, x, 1, z_{4}\right) .
$$

Solving these two equations we find

$$
\hat{A}(x, \bar{x})=x(1-x) \frac{\partial}{\partial x} \ln I(x, \bar{x})
$$

with

$$
\begin{aligned}
I(x, \bar{x})=\mid & 1-\left.x\right|^{\frac{2}{N}\left(k_{3}-k_{2}\right)} \frac{\Gamma\left(1-\frac{k_{1}}{N}\right) \Gamma\left(\frac{k_{2}}{N}\right) \Gamma\left(\frac{k_{3}}{N}\right) \Gamma\left(1-\frac{k_{2}}{N}\right)}{\Gamma\left(1-\frac{k_{1}}{N}+\frac{k_{2}}{N}\right) \Gamma\left(1-\frac{k_{2}}{N}+\frac{k_{3}}{N}\right)} F_{1}(x) \bar{G}_{2}(1-\bar{x}) \\
& +|x|^{\frac{2}{N}\left(k_{1}-k_{2}\right)} \frac{\Gamma\left(1-\frac{k_{3}}{N}\right) \Gamma\left(\frac{k_{2}}{N}\right) \Gamma\left(\frac{k_{1}}{N}\right) \Gamma\left(1-\frac{k_{2}}{N}\right)}{\Gamma\left(1-\frac{k_{3}}{N}+\frac{k_{2}}{N}\right) \Gamma\left(1-\frac{k_{2}}{N}+\frac{k_{1}}{N}\right)} F_{2}(1-x) \bar{G}_{1}(\bar{x}),
\end{aligned}
$$

and

$$
\begin{aligned}
& \hat{B}(x, \bar{x})=x(1-x) \frac{\Gamma\left(1-\frac{k_{1}}{N}\right) \Gamma\left(\frac{k_{2}}{N}\right) \Gamma\left(1-\frac{k_{3}}{N}\right) \Gamma\left(\frac{k_{2}}{N}\right)}{\Gamma\left(1-\frac{k_{1}}{N}+\frac{k_{2}}{N}\right) \Gamma\left(1-\frac{k_{3}}{N}+\frac{k_{2}}{N}\right)} I^{-1}(x, \bar{x}) \\
& \times\left\{(1-x)^{\frac{k_{2}}{N}-\frac{k_{3}}{N}} F_{2}(1-x) \partial_{x}\left[(1-x)^{\frac{k_{3}}{N}-\frac{k_{2}}{N}} F_{1}(x)\right]-x^{\frac{k_{2}}{N}-\frac{k_{1}}{N}} F_{1}(x) \partial_{x}\left[x^{\frac{k_{1}}{N}-\frac{k_{2}}{N}} F_{2}(1-x)\right]\right\} .
\end{aligned}
$$


During the computation, we utilized the formula A.3. Inserting these functions $\hat{A}(x, \bar{x})$ and $\hat{B}(x, \bar{x})$, we have the explicit form of the green functions $g(z, w ; x, \bar{x})(4.6)$ and $h(\bar{z}, w ; x, \bar{x})(4.7)$. The other two green functions $\bar{g}(\bar{z}, \bar{w} ; x, \bar{x})$ and $\bar{g}(z, \bar{w} ; x, \bar{x})$ are given by replacing the arguments with bar and without bar and $k_{i} \leftrightarrow N-k_{i}$.

From the expression of $\hat{A}(x, \bar{x})$, we can read off the four point function (4.5) as follows. A limit of the green function (4.6) gives the correlation function involving the energy momentum tensor as

$$
\lim _{z \rightarrow w}\left[g(z, w)-\frac{1}{(z-w)^{2}}\right]=\frac{\left\langle T(z) \prod_{i=1}^{4} \sigma_{k_{i}}^{\epsilon_{i}}\left(z_{i}, \bar{z}_{i}\right)\right\rangle}{\left\langle\prod_{i=1}^{4} \sigma_{k_{i}}^{\epsilon_{i}}\left(z_{i}, \bar{z}_{i}\right)\right\rangle} .
$$

Since the OPE with the energy momentum tensor reads ${ }^{7}$

$$
T(z) \sigma_{k_{2}}^{+}\left(z_{2}\right) \sim \frac{h_{2}}{\left(z-z_{2}\right)^{2}}+\frac{\partial_{z_{2}} \sigma_{k_{2}}\left(z_{2}\right)}{z-z_{2}}
$$

we can obtain from the correlation function (4.28) as

$$
\partial_{z_{2}} \ln Z_{N}\left(z_{i}, \bar{z}_{i}\right)=\frac{A\left(z_{i}, \bar{z}_{i}\right)}{\left(z_{2}-z_{1}\right)\left(z_{2}-z_{3}\right)\left(z_{2}-z_{4}\right)}-\frac{\left(1-\frac{k_{1}}{N}\right) \frac{k_{2}}{N}}{z_{2}-z_{1}}-\frac{\left(1-\frac{k_{3}}{N}\right) \frac{k_{2}}{N}}{z_{2}-z_{3}}-\frac{\frac{k_{2}}{N} \frac{k_{4}}{N}}{z_{2}-z_{4}} .
$$

Setting $\left(z_{1}, z_{2}, z_{3}, z_{4}\right)=(0, x, 1, \infty)$ and integrating by $x$, we find

$$
Z_{N}(x, \bar{x})=\mathcal{N}|x|^{-\frac{2 k_{2}}{N}\left(1-\frac{k_{1}}{N}\right)}|1-x|^{-\frac{2 k_{2}}{N}\left(1-\frac{k_{3}}{N}\right)} I^{-1}(x, \bar{x})
$$

with a constant $\mathcal{N}$, which will be fixed later. Here we have defined

$$
Z_{N}(x, \bar{x})=\lim _{z_{4} \rightarrow \infty}\left|-z_{4}^{2}\right|^{h_{4}} Z_{N}\left(z_{i}, \bar{z}_{i}\right)
$$

In this way, we have obtained the explicit form of the four point function without excitation (4.5) as (4.31).

Now that we have the explicit form of the green functions (4.6), (4.7) and the four point function (4.5), it is easy to write down explicitly the general four point function through (4.11). We only need to compute the limit like (4.9). The one in (4.9) is given by

$$
\begin{aligned}
& g^{\prime}\left(z_{2}, z_{1} ; z_{i}, \bar{z}_{i}\right)=e^{\pi i\left(-1+\frac{k_{1}}{N}\right)}\left(z_{2}-z_{1}\right)^{-\frac{k_{1}}{N}}\left(z_{2}-z_{3}\right)^{-\frac{k_{3}}{N}}\left(z_{2}-z_{4}\right)^{-1+\frac{k_{4}}{N}} \\
& \quad \times\left(z_{1}-z_{2}\right)^{-\frac{k_{2}}{N}}\left(z_{1}-z_{3}\right)^{-1+\frac{k_{3}}{N}}\left(z_{1}-z_{4}\right)^{-\frac{k_{4}}{N}}\left[A\left(z_{i}, \bar{z}_{i}\right)+\frac{k_{2}}{N}\left(z_{3}-z_{2}\right)\left(z_{2}-z_{4}\right)\right] \\
& \quad=e^{\pi i \frac{k_{4}}{N}} x^{-\frac{k_{1}}{N}-\frac{k_{2}}{N}}(x-1)^{-\frac{k_{3}}{N}}\left[\hat{A}(x, \bar{x})+\frac{k_{2}}{N}(1-x)\right] .
\end{aligned}
$$

\footnotetext{
${ }^{7}$ We denote $h_{i}\left(=h_{k_{i}}\right)=\frac{1}{2} \frac{k_{i}}{N}\left(1-\frac{k_{i}}{N}\right)$.
} 
In the last equation, we fix the positions as $\left(z_{1}, z_{2}, z_{3}, z_{4}\right)=(0, x, 1, \infty)$. In the same way, we obtain

$$
g^{\prime}\left(z_{2}, z_{3}\right)=-x^{-\frac{k_{1}}{N}}(1-x)^{-\frac{k_{2}}{N}-\frac{k_{3}}{N}}\left[\hat{A}(x, \bar{x})-\frac{k_{2}}{N} x\right] .
$$

When we take the limit of $z \rightarrow z_{4}$ and also $z_{4} \rightarrow \infty$, we need to define as

$$
\begin{aligned}
g^{\prime}\left(z_{4}, z_{1} ; x, \bar{x}\right) & =\lim _{z_{4} \rightarrow \infty}\left(-z_{4}^{2}\right)^{\frac{k_{4}}{N}} g^{\prime}\left(z_{4}, z_{1} ; 0, x, 1, z_{4}\right) \\
& =-e^{\pi i \frac{k_{4}}{N}} x^{-\frac{k_{2}}{N}}\left[\hat{A}(x, \bar{x})-\frac{k_{1}}{N}+\frac{k_{2}}{N}-\frac{k_{2}}{N} x\right], \\
g^{\prime}\left(z_{4}, z_{3} ; x, \bar{x}\right) & =\lim _{z_{4} \rightarrow \infty}\left(-z_{4}^{2}\right)^{\frac{k_{4}}{N}} g^{\prime}\left(z_{4}, z_{3} ; 0, x, 1, z_{4}\right) \\
& =e^{\pi i \frac{k_{3}}{N}}(1-x)^{-\frac{k_{2}}{N}}\left[\hat{A}(x, \bar{x})+\frac{k_{3}}{N}-\frac{k_{2}}{N} x\right] .
\end{aligned}
$$

For the green function $h(\bar{z}, w)(4.6)$, we similarly obtain

$$
\begin{aligned}
& h^{\prime}\left(\bar{z}_{1}, z_{1}\right)=|x|^{-\frac{2 k_{2}}{N} \hat{B}}(x, \bar{x}), \quad h^{\prime}\left(\bar{z}_{1}, z_{3}\right)=e^{-\pi i\left(-1+\frac{k_{3}}{N}\right)}[\bar{x}(x-1)]^{-\frac{2 k_{2}}{N}} \hat{B}(x, \bar{x}), \\
& h^{\prime}\left(\bar{z}_{1}, z_{1}\right)=e^{\pi i\left(-1+\frac{k_{3}}{N}\right)}[x(\bar{x}-1)]^{-\frac{2 k_{2}}{N}} \hat{B}(x, \bar{x}), \quad h^{\prime}\left(\bar{z}_{3}, z_{3}\right)=|1-x|^{-\frac{2 k_{2}}{N}} \hat{B}(x, \bar{x}) .
\end{aligned}
$$

In the next two subsections, we will read off the three point functions from the factorization, where the limits $x \rightarrow 0$ and $x \rightarrow \infty$ of the above green functions are taken.

\subsection{Three point functions of excited twist fields}

The four point function can be factorized by the product of three point functions when expanding around $x \sim 0$ or $x \sim \infty$ (and also $x \sim 1$ ) as explained before. We begin with the four point function without excitation (4.5). First we examine the factorization around $x, \bar{x} \sim 0$, where we should use the OPE between the twist fields $\sigma_{k_{1}}^{-}$and $\sigma_{k_{2}}^{+}$. Suppose $k_{1}-k_{2}=-k_{3}+k_{4}=k_{I}>0$, where the twist number of the intermediate field $\sigma_{k_{I}}^{-}$is set by the twist number conservation. Then, we can use the OPEs as

$$
\begin{aligned}
\sigma_{k_{1}}^{-}(z, \bar{z}) \sigma_{k_{2}}^{+}(w, \bar{w}) & \sim \frac{C_{(-, 1)(+, 2)}^{(-, I)} \sigma_{k_{I}}^{-}(w, \bar{w})}{|z-w|^{2 h_{1}+2 h_{2}-2 h_{I}}}, \\
\sigma_{k_{3}}^{-}(z, \bar{z}) \sigma_{k_{4}}^{+}(w, \bar{w}) & \sim \frac{C_{(-, 3)(+, 4)}^{(+, I)} \sigma_{k_{I}}^{+}(w, \bar{w})}{|z-w|^{2 h_{3}+2 h_{4}-2 h_{I}}},
\end{aligned}
$$

which leads to the factorization of the four point function as

$$
Z_{N}(x, \bar{x}) \sim|x|^{-2 h_{1}-2 h_{2}+2 h_{I}} C_{(-, 1)(+, 2)}^{(-, I)} C_{(-, 3)(+, 4)(-, I)} .
$$


Here we should note that the index is raised or lowered by the two point function $\left\langle\sigma_{k}^{-}(\infty) \sigma_{k^{\prime}}^{+}(0)\right\rangle=\delta_{k, k^{\prime}}$.

Since the four point function is written in terms of $I(x, \bar{x})$ as in $(4.31)$, we can read off the three point functions (4.38) from the asymptotic behavior of $I(x, \bar{x})$ around $x, \bar{x} \sim 0$

$$
\begin{aligned}
I(x, \bar{x}) \sim & \frac{\Gamma\left(1-\frac{k_{1}}{N}\right) \Gamma\left(\frac{k_{2}}{N}\right) \Gamma\left(\frac{k_{3}}{N}\right) \Gamma\left(\frac{k_{1}}{N}-\frac{k_{2}}{N}\right)}{\Gamma\left(\frac{k_{4}}{N}\right) \Gamma\left(1+\frac{k_{2}}{N}-\frac{k_{1}}{N}\right)} \\
& +\frac{\Gamma\left(1-\frac{k_{3}}{N}\right) \Gamma\left(\frac{k_{1}}{N}\right) \Gamma\left(1-\frac{k_{2}}{N}\right) \Gamma\left(\frac{k_{2}}{N}-\frac{k_{1}}{N}\right)}{\Gamma\left(1-\frac{k_{4}}{N}\right) \Gamma\left(1-\frac{k_{2}}{N}+\frac{k_{1}}{N}\right)}|x|^{\frac{2}{N}\left(k_{1}-k_{2}\right)} .
\end{aligned}
$$

For later convenience, we rewrite the normalization $\mathcal{N}$ as

$$
\mathcal{N}=v \sqrt{\frac{\pi^{2} \sin \frac{\pi k_{4}}{N}}{\sin \frac{\pi k_{1}}{N} \sin \frac{\pi k_{2}}{N} \sin \frac{\pi k_{3}}{N}}}
$$

then the three point functions (4.38) are given as

$$
C_{(-, 1)(+, 2)(+, I)}=\sqrt{v \frac{\gamma\left(\frac{k_{1}}{N}\right)}{\gamma\left(\frac{k_{2}}{N}\right) \gamma\left(\frac{k_{I}}{N}\right)}}, \quad C_{(-, 3)(+, 4)(-, I)}=\sqrt{v \frac{\gamma\left(\frac{k_{4}}{N}\right)}{\gamma\left(\frac{k_{3}}{N}\right) \gamma\left(\frac{k_{I}}{N}\right)}},
$$

where we have used the notation $\gamma(x)=\Gamma(x) / \Gamma(1-x)$. In the large $N$ limit, we reproduce the classical expression (3.18) with the help of the relation

$$
\frac{\gamma\left(\frac{k_{1}}{N}\right)}{\gamma\left(\frac{k_{2}}{N}\right) \gamma\left(\frac{k_{I}}{N}\right)} \sim \frac{\frac{k_{2}}{N} \frac{k_{I}}{N}}{\frac{k_{1}}{N}}
$$

For $k_{2}-k_{1}=-k_{4}+k_{3}=k_{I}>0$, we can similarly obtain the factorization as

$$
Z_{N}(x, \bar{x})=|x|^{-2 h_{1}-2 h_{2}+2 h_{I}} C_{(-, 1)(+, 2)}^{(+, I)} C_{(-, 3)(+, 4)(+, I)} .
$$

The three point functions are also read from the asymptotic behavior of $I(x, \bar{x})$ as

$$
C_{(-, 1)(+, 2)(-, I)}=\sqrt{v \frac{\gamma\left(\frac{k_{2}}{N}\right)}{\gamma\left(\frac{k_{1}}{N}\right) \gamma\left(\frac{k_{I}}{N}\right)}}, \quad C_{(-, 3)(+, 4)(+, I)}=\sqrt{v \frac{\gamma\left(\frac{k_{3}}{N}\right)}{\gamma\left(\frac{k_{I}}{N}\right) \gamma\left(\frac{k_{4}}{N}\right)}},
$$

which are the same as the previous ones (4.42). Since the intermediate field is in the untwisted sector for $k_{2}-k_{1}=0$, we will discuss it separately in the next subsection.

We can also obtain similar factorization for $x, \bar{x} \sim \infty$, where the OPE between $\sigma_{k_{2}}^{+}$ and $\sigma_{k_{4}}^{+}$is used. Since the function $I(x, \bar{x})$ has the asymptotic behavior

$$
I(x, \bar{x}) \sim|x|^{-\frac{2 k_{2}}{N}} \frac{\Gamma\left(\frac{k_{1}}{N}\right) \Gamma\left(\frac{k_{2}}{N}\right) \Gamma\left(\frac{k_{3}}{N}\right) \Gamma\left(1-\frac{k_{1}}{N}-\frac{k_{3}}{N}\right)}{\Gamma\left(1-\frac{k_{4}}{N}\right) \Gamma\left(\frac{k_{1}}{N}+\frac{k_{3}}{N}\right)},
$$

the four point function (4.5) is factorized as

$$
\left|-x^{2}\right|^{2 h_{2}} Z_{N}(x, \bar{x}) \sim\left|\frac{1}{x}\right|^{-2 h_{2}-2 h_{4}+2 h_{I}} C_{(+, 2)(+, 4)}^{(+, I)} C_{(-, 1)(-, 3)(+, I)}
$$


with $k_{I}=k_{2}+k_{4}=k_{1}+k_{3}$, and the three point functions are read as

$$
C_{(+, 2)(+, 4)(-, I)}=\sqrt{v \frac{\gamma\left(\frac{k_{I}}{N}\right)}{\gamma\left(\frac{k_{2}}{N}\right) \gamma\left(\frac{k_{4}}{N}\right)}}, \quad C_{(-, 1)(-, 3)(+, I)}=\sqrt{v \frac{\gamma\left(\frac{k_{I}}{N}\right)}{\gamma\left(\frac{k_{1}}{N}\right) \gamma\left(\frac{k_{3}}{N}\right)}} .
$$

These results reproduce (4.42) obtained for $x, \bar{x} \sim 0$ as well.

We move to include excitations, namely, to compute the quantum version of three point function (3.26). We start from the case of (3.22), which can be deduced from the four point function without excitation (4.5). Since the asymptotic behavior of the function $I(x, \bar{x})$ is given by $(4.40)$, non-trivial corrections are written for $k_{1}-k_{2}=k_{I}>0$ as

$$
Z_{N}(x, \bar{x}) \sim \sum_{n=0}|x|^{-2 h_{1}-2 h_{2}+2 h_{I}+\frac{2 n k_{I}}{N}} C_{(-, 1)(+, 2)}^{(-, I, n, n)} C_{(-, 3)(+, 4)(-, I, n, n)}
$$

where the three point functions are

$$
\begin{aligned}
& C_{(-, 1)(+, 2)(+, I, n, n)}=n !\left(\epsilon \frac{\gamma\left(\frac{k_{1}}{N}\right)}{\gamma\left(\frac{k_{2}}{N}\right) \gamma\left(\frac{k_{I}}{N}\right)}\right)^{n} C_{(-, 1)(+, 2)(+, I)}, \\
& C_{(-, 3)(+, 4)(-, I, n, n)}=n !\left(\epsilon \frac{\gamma\left(\frac{k_{4}}{N}\right)}{\gamma\left(\frac{k_{3}}{N}\right) \gamma\left(\frac{k_{I}}{N}\right)}\right)^{n} C_{(-, 3)(+, 4)(-, I)} .
\end{aligned}
$$

The index $(+, I, n, n)$ means that the intermediate field is an excited field $\sigma_{k_{I}}^{+, n, n}$, whose normalization is given by (3.4). The phase factor $\epsilon= \pm 1$ cannot be determined here, so we will fix it below in another way of factorization. This expression reproduces the classical one (3.22) if we use $\epsilon=+1$ and (4.43). We can obtain the similar result for $k_{2}-k_{1}>0$.

In order to deal with other types of excitation, we have to consider the four point functions with quasi-zero modes. First we consider the correlation function

$$
Z_{N}=\left\langle\sigma_{k_{1}}^{-, n, 0}\left(z_{1}, \bar{z}_{1}\right) \sigma_{k_{2}}^{+, n, 0}\left(z_{2}, \bar{z}_{2}\right) \sigma_{k_{3}}^{-}\left(z_{3}, \bar{z}_{3}\right) \sigma_{k_{4}}^{+}\left(z_{4}, \bar{z}_{4}\right)\right\rangle_{N}
$$

where we include $\left(i \partial X^{+}(z)\right)^{n}$ and $\left(i \partial X^{-}(w)\right)^{n}$ in the four point function (4.5) and take the limits $z \rightarrow z_{2}$ and $w \rightarrow z_{1}$. Setting $\left(z_{1}, z_{2}, z_{3}, z_{4}\right)=(0, x, 1, \infty)$ and taking the limit $x, \bar{x} \sim 0$, we can obtain the quantum counterpart of (3.20) through the factorization as follows. Since the limit of green function is given in (4.33), the function behaves for $k_{1}-k_{2}=k_{I}>0$ as

$$
g^{\prime}\left(z_{2}, z_{1}\right) \sim e^{\pi i\left(\frac{k_{1}}{N}-\frac{k_{2}}{N}\right)} x^{-\frac{k_{1}}{N}-\frac{k_{2}}{N} \frac{k_{2}}{N}}
$$

Therefore, the above correlator may be factorized as

$$
Z_{N} \sim x^{-\frac{n k_{1}}{N}-\frac{n k_{2}}{N}}|x|^{-2 h_{1}-2 h_{2}+2 h_{I}} C_{(-, 1, n, 0)(+, 2, n, 0)}^{(-, I)} C_{(-, 3)(+, 4)(-, I)},
$$


where the three point function is 8

$$
C_{(-, 1, n, 0)(+, 2, n, 0)(+, I)}=n !\left(\frac{k_{2}}{N}\right)^{n} C_{(-, 1)(+, 2)(+, I)} .
$$

This result means that the classical result (3.20) does not receive any quantum corrections. Similarly, for $k_{2}-k_{1}=k_{I}>0$, we find

$$
Z_{N} \sim x^{-\frac{n k_{1}}{N}-\frac{n k_{2}}{N}}|x|^{-2 h_{1}-2 h_{2}+2 h_{I}} C_{(-, 1, n, 0)(+, 2, n, 0)}^{(+, I)} C_{(-, 3)(+, 4)(+, I)},
$$

where the three point function is

$$
C_{(-, 1, n, 0)(+, 2, n, 0)(-, I)}=n !\left(\frac{k_{1}}{N}\right)^{n} C_{(-, 1)(+, 2)(-, I)} .
$$

This also reproduces (3.20). We can obtain the same result even if we use the limit of $x, \bar{x} \sim \infty$. In that case, however, we cannot fix the phase factor $\epsilon= \pm 1$ as in (4.50).

Next we compute the quantum three point functions of the type (3.22) to fix the phase factor in (4.50). The case of (3.21) follows soon. For that purpose, it is convenient to consider the four point function

$$
Z_{N}=\left\langle\sigma_{k_{1}}^{-, n, n}\left(z_{1}, \bar{z}_{1}\right) \sigma_{k_{2}}^{+}\left(z_{2}, \bar{z}_{2}\right) \sigma_{k_{3}}^{-}\left(z_{3}, \bar{z}_{3}\right) \sigma_{k_{4}}^{+}\left(z_{4}, \bar{z}_{4}\right)\right\rangle_{N},
$$

and take $x, \bar{x} \sim \infty$ with $\left(z_{1}, z_{2}, z_{3}, z_{4}\right)=(0, x, 1, \infty)$. After some computation, we obtain the limit of the green function (4.37)

$$
h^{\prime}\left(\bar{z}_{1}, z_{1}\right)=|x|^{-\frac{2 k_{2}}{N}} \hat{B}(x, \bar{x}), \quad \hat{B}(x, \bar{x}) \sim x(x-1)|x|^{\frac{2 k_{2}}{N}} \frac{\gamma\left(\frac{k_{I}}{N}\right)}{\gamma\left(\frac{k_{3}}{N}\right) \gamma\left(\frac{k_{1}}{N}\right)} x^{-2}
$$

with $k_{I}=k_{1}+k_{3}=k_{2}+k_{4}$. Therefore, the four point function is factorized into

$$
\left|-x^{2}\right|^{2 h_{2}} Z_{N} \sim\left|\frac{1}{x}\right|^{-2 h_{2}-2 h_{4}+2 h_{I}} C_{(+, 2)(+, 4)}^{(+, I)} C_{(-, 1, n, n)(-, 3)(+, I)}
$$

with the three point function

$$
C_{(-, 1, n, n)(-, 3)(+, I)}=n !\left(\frac{\gamma\left(\frac{k_{I}}{N}\right)}{\gamma\left(\frac{k_{1}}{N}\right) \gamma\left(\frac{k_{3}}{N}\right)}\right)^{n} C_{(-, 1)(-, 3)(+, I)} .
$$

Notice that we can fix the phase factor in (4.50) as $\epsilon=1$.

The other three point function of the type (3.21) can be obtained in a similar way. Here we consider

$$
Z_{N}=\left\langle\sigma_{k_{1}}^{-, n, 0}\left(z_{1}, \bar{z}_{1}\right) \sigma_{k_{2}}^{+}\left(z_{2}, \bar{z}_{2}\right) \sigma_{k_{3}}^{-, 0, n}\left(z_{3}, \bar{z}_{3}\right) \sigma_{k_{4}}^{+}\left(z_{4}, \bar{z}_{4}\right)\right\rangle
$$

${ }^{8}$ We will neglect the phase factor like $e^{\pi i\left(\frac{k_{1}}{N}-\frac{k_{2}}{N}\right)}$ in the following. This phase factor arises when we insert $\left(z_{1}, z_{2}, z_{3}, z_{4}\right)=(0, x, 1, \infty)$ in the factorization formula (4.14) if the conformal weights do not match for holomorphic and anti-holomorphic parts. 
and take $x, \bar{x} \sim \infty$ with $\left(z_{1}, z_{2}, z_{3}, z_{4}\right)=(0, x, 1, \infty)$. Making use of the behavior of the green function involved (4.37)

$$
h^{\prime}\left(\bar{z}_{1}, z_{3}\right) \sim-|x|^{-\frac{2 k_{2}}{N}} \hat{B}(x, \bar{x}), \quad \hat{B}(x, \bar{x}) \sim|x|^{\frac{2 k_{2}}{N}} \frac{\gamma\left(\frac{k_{I}}{N}\right)}{\gamma\left(\frac{k_{3}}{N}\right) \gamma\left(\frac{k_{1}}{N}\right)},
$$

the four point function can be factorized as

$$
\left|-x^{2}\right|^{2 h_{2}} Z_{N} \sim\left|\frac{1}{x}\right|^{-2 h_{2}-2 h_{4}+2 h_{I}} C_{(+, 2)(+, 4)}^{(+, I)} C_{(-, 1, n, 0)(-, 3,0, n)(+, I)}
$$

with the three point function

$$
C_{(-, 1, n, 0)(-, 3,0, n)(+, I)}=n !\left(-\frac{\gamma\left(\frac{k_{I}}{N}\right)}{\gamma\left(\frac{k_{1}}{N}\right) \gamma\left(\frac{k_{3}}{N}\right)}\right)^{n} C_{(-, 1)(-, 3)(+, I)} .
$$

This reproduces the classical result (3.21) if we apply (4.43).

Now we have every non-trivial three point functions with the excitation of quasi-zero mode. In the basis of (2.30), we can express for $k_{1}=k_{2}+k_{3}$ as

$$
\begin{aligned}
& { }_{N}\left\langle y_{1}, \tilde{y}_{1}, \mp k_{1}\left|\sigma_{k_{2}}^{ \pm, y_{2}, \tilde{y}_{2}}\right| y_{3}, \tilde{y}_{3}, \pm k_{3}\right\rangle_{N} \\
& \quad=e^{y_{3}\left(\frac{k_{1}}{N} y_{1}+\frac{k_{2}}{N} y_{2}\right)+\tilde{y}_{3}\left(\frac{k_{1}}{N} \tilde{y}_{1}+\frac{k_{2}}{N} \tilde{y}_{2}\right)+\frac{\gamma\left(\frac{k_{1}}{N}\right)}{\gamma\left(\frac{k_{2}}{N}\right) \gamma\left(\frac{k_{3}}{N}\right)}\left(y_{2}-y_{3}\right)\left(\tilde{y}_{2}-\tilde{y}_{3}\right)} C_{(-, 1)(+, 2)(+, 3)} .
\end{aligned}
$$

This expression coincides with the one in [11], where they applied an analytic continuation to the result of Nappi-Witten model [36, 37, 38]. Our method does not only reproduce their results, but gives a way to compute more generic correlation functions. For instance, we have obtained a generic four point function in (4.11). The other correlation functions can be computed by following our method.

\subsection{Three point functions with an untwisted field}

In this section, we rederive the three point functions with two twisted states and one untwisted state. The correlation functions were already obtained in subsection 3.1 in operator formalism, but we will show that our method correctly reproduces the results. We consider the four point function (4.5) without excitation for $k_{1}=k_{2}=k$ and $k_{3}=k_{4}=l$

and take the limit of $x, \bar{x} \sim 0$. Here we should note that the asymptotic behavior of the hypergeometric functions becomes

$$
\begin{aligned}
& F_{2}(1-x) \sim \frac{\Gamma\left(1-\frac{l}{N}+\frac{k}{N}\right)}{\Gamma\left(\frac{k}{N}\right) \Gamma\left(1-\frac{l}{N}\right)}\left[2 \psi(1)-\psi\left(\frac{k}{N}\right)-\psi\left(1-\frac{l}{N}\right)-\ln x\right] \\
& \bar{G}_{2}(1-\bar{x}) \sim \frac{\Gamma\left(1-\frac{k}{N}+\frac{l}{N}\right)}{\Gamma\left(\frac{l}{N}\right) \Gamma\left(1-\frac{k}{N}\right)}\left[2 \psi(1)-\psi\left(\frac{l}{N}\right)-\psi\left(1-\frac{k}{N}\right)-\ln \bar{x}\right]
\end{aligned}
$$


and hence the function $I(x, \bar{x})$ behaves as

$$
I(x, \bar{x}) \sim \frac{\pi}{\sin \frac{\pi k}{N}} \ln \frac{\delta_{k} \delta_{l}}{|x|^{2}},
$$

where we have used the notation of $\delta_{n}$ in (3.9). Using a formula

$$
\left(-2 \ln \frac{|x|}{\sqrt{\delta_{k} \delta_{l}}}\right)^{-n-1}=\frac{1}{2 \pi n !} \int d p d \bar{p}(p \bar{p})^{n}\left(\frac{|x|}{\sqrt{\delta_{k} \delta_{l}}}\right)^{2 p \bar{p}}
$$

we find

$$
Z_{N}(x, \bar{x})=v \frac{|x|^{-4 h_{k}}}{-2 \ln \frac{|x|}{\sqrt{\delta_{k} \delta_{l}}}}=|x|^{-4 h_{k}} \int \frac{d p d \bar{p}}{(2 \pi)^{2}}\left(\frac{|x|}{\sqrt{\delta_{k} \delta_{l}}}\right)^{2 p \bar{p}} .
$$

The above equation (4.69) implies that the three point functions are

$$
C_{(-, k)(+, k) p}=\delta_{k}^{-p \bar{p}}, \quad C_{(-, l)(+, l) p}=\delta_{l}^{-p \bar{p}} .
$$

The normalization is set $\operatorname{as}^{9} v=\frac{1}{2 \pi}$ such that $C_{(-, k)(+, k) 0}=1$. Here we should recall that in the untwisted sector we can map the basis of the flat space into the orbifold one as in subsection 2.1 .

We then include the excitation of quasi-zero modes. We first compute the four point function

$$
Z_{N}=\left\langle\sigma_{k}^{-}\left(z_{1}, \bar{z}_{1}\right) \sigma_{k}^{+, m, 0}\left(z_{2}, \bar{z}_{2}\right) \sigma_{l}^{-, m, 0}\left(z_{3}, \bar{z}_{3}\right) \sigma_{l}^{+}\left(z_{4}, \bar{z}_{4}\right)\right\rangle_{N}
$$

and take the limit of $x, \bar{x} \sim 0$ with $\left(z_{1}, z_{2}, z_{3}, z_{4}\right)=(0, x, 1, \infty)$. We utilize the green function (4.34), which behaves as

$$
g^{\prime}\left(z_{2}, z_{3}\right)=-(-1)^{\frac{l}{N}} x^{-\frac{k}{N}}(1-x)^{-\frac{k}{N}-\frac{l}{N}}\left[\hat{A}(x, \bar{x})-\frac{k}{N} x\right], \quad A(x, \bar{x}) \sim \frac{1}{-\ln \frac{\delta_{k} \delta_{l}}{|x|^{2}}} .
$$

With the help of this behavior and the formula (4.68), we can obtain the factorization as

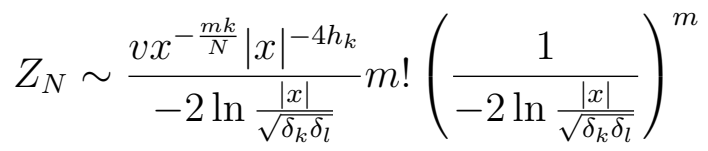

$$
\begin{aligned}
& =x^{-\frac{m k}{N}}|x|^{-4 h_{k}} \int \frac{d p d \bar{p}}{(2 \pi)^{2}} C_{(-, k)(+, k, m, 0)}^{p} C_{(-, l, m, 0)(+, l) p},
\end{aligned}
$$

where the three point functions are ${ }^{10}$

$$
C_{(-, k)(+, k, m, 0) p}=(-p)^{m} \delta_{k}^{-p \bar{p}}, \quad C_{(-, l, m, 0)(+, l) p}=(-\bar{p})^{m} \delta_{l}^{-p \bar{p}}
$$

${ }^{9}$ This does not contradict the classical result (3.18) where $v=1 / \pi$ in this notation. This can be seen from the fact that it takes $v=\frac{1}{\alpha^{\prime} \pi}$ if $\alpha^{\prime}=2$ is written explicitly.

${ }^{10}$ The phase factor cannot be fixed only from the factorization. We use the one consistent with the result in operator formalism. 
We next compute a more generic four point function

$$
Z_{N}=\left\langle\sigma_{k}^{-, n, 0}\left(z_{1}, \bar{z}_{1}\right) \sigma_{k}^{+, n+m, 0}\left(z_{2}, \bar{z}_{2}\right) \sigma_{l}^{-, m, 0}\left(z_{3}, \bar{z}_{3}\right) \sigma_{l}^{+}\left(z_{4}, \bar{z}_{4}\right)\right\rangle_{N}
$$

and take the limit of $x, \bar{x} \sim 0$ with $\left(z_{1}, z_{2}, z_{3}, z_{4}\right)=(0, x, 1, \infty)$. Here we use the limit of the green function (4.33) as well. From the similar computation, we obtain

$$
\begin{aligned}
Z_{N} & \sim \frac{v x^{-\frac{(2 n+m) k}{N}}|x|^{-4 h_{k}}}{-2 \ln \frac{|x|}{\sqrt{\delta_{k} \delta_{l}}}}(n+m) !\left(\frac{k}{N}-\frac{1}{-2 \ln \frac{|x|}{\sqrt{\delta_{k} \delta_{l}}}}\right)^{n}\left(\frac{1}{-2 \ln \frac{|x|}{\sqrt{\delta_{k} \delta_{l}}}}\right)^{m} \\
& =x^{-\frac{(2 n+m) k}{N}}|x|^{-4 h_{k}} \int \frac{d p d \bar{p}}{(2 \pi)^{2}}|x|^{2 p \bar{p}} C_{(-, k, n, 0)(+, k, n+m, 0)}^{p} C_{(-, l, m, 0)(+, k) p}
\end{aligned}
$$

with the three point function

$$
C_{(-, k, n, 0)(+, k, n+m, 0) p}=(n+m) !\left(\frac{k}{N}\right)^{n+m} \bar{p}^{-m} L_{n+m}^{(-m)}\left(\frac{N p \bar{p}}{k}\right) \delta_{k}^{-p \bar{p}}
$$

The definition of generalized Laguerre polynomial

$$
L_{n}^{(m)}(x)=\sum_{l=0}^{n}(-1)^{l}\left(\begin{array}{c}
n+m \\
n-l
\end{array}\right) \frac{x^{l}}{l !}
$$

was used in the above coefficient.

In the similar manner, we can show that the three point functions of the type like $C_{(-, k, n, \bar{n})(+, k) p}$ vanishes from the behavior of the green function $h^{\prime}\left(\bar{z}_{1}, z_{1}\right)$. Combined with the anti-holomorphic part, we therefore conclude that

$$
{ }_{N}\left\langle y_{1}, \tilde{y}_{1}, k\left|: e^{i \bar{p} X^{+}+i p X^{-}}:\right| y_{2}, \tilde{y}_{2}, k\right\rangle_{N}=e^{\frac{k}{N}\left(y_{1} y_{2}+\tilde{y}_{1} \tilde{y}_{2}\right)-\bar{p}\left(y_{1}-\tilde{y}_{2}\right)+p\left(y_{2}-\tilde{y}_{1}\right)}
$$

which reproduces the result in operator formalism (3.13). The generating function for the generalized Laguerre polynomial

$$
e^{x y+r(x-y)}=\sum_{n, l=0}^{\infty} \frac{r^{n-l}}{l !} L_{l}^{(n-l)}\left(r^{2}\right) x^{n} y^{l}
$$

was used to summarize in the above form.

\section{Conclusion and discussions}

In this paper, we have developed a general method to compute correlation functions in Misner space. Even though the correlators with less than two twist fields can be computed in operator formalism as in subsection 3.1, we need a general technique to compute those with more twist fields. In order to perform the path integral to compute correlation functions, we may have to deal with an Euclidean target space. We therefore perform the 
Wick rotation to the Lorentzian orbifold, and relate to the $\mathbb{C} / \mathbb{Z}_{N}$ orbifold theory. It is a subtle problem to determine the spectrum in Misner space, and we utilized the Mellin transformation (2.29) to construct a map from the spectrum in the $\mathbb{C} / \mathbb{Z}_{N}$ orbifold. The general method to compute correlators in the $\mathbb{C} / \mathbb{Z}_{N}$ orbifold was already developed in $[43,44]$ by making use of the monodromy conditions. We have computed a general four point function (4.11), where the excitation of quasi-zero modes are included by utilizing the green functions in the presence of four twist fields. The three point functions have been read off from the four point function through the factorization, and we obtained (4.65) for those of twist fields and (4.80) for those with an untwisted field.

A motivation to compute the correlation functions is to investigate the role of winding strings in Misner space, in particular, the relation to the (possible) resolution of the big crunch/big bang singularity. Let us first see the condensation of tachyonic strings wrapped on the whole whisker regions (2.28). From the viewpoint of worldsheet theory, the condensation is described by the deformation of twist fields

$$
S=S_{0}+\alpha_{k} \int d^{2} z V_{k}+\alpha_{-k} \int d^{2} z V_{-k}
$$

where $V_{ \pm k}$ denote the twist fields corresponding to the winding tachyon fields. The change of geometry may be read from the graviton scattering

$$
\left\langle G_{\mu \nu} G^{\mu \nu}\right\rangle_{\alpha}=\left\langle G_{\mu \nu} G^{\mu \nu}\right\rangle_{0}+\left|\alpha_{k}\right|^{2}\left\langle V_{-k} G_{\mu \nu} G^{\mu \nu} V_{k}\right\rangle_{0}+\cdots,
$$

where the correlators are given by the overlaps of the corresponding wave functions with the stringy correction like in (3.13). Therefore, in the region where the tachyon fields are localized, the graviton modes are frozen out due to the large mass term or the deformation of the background geometry. Since the tachyon fields are wrapped over the whisker regions, the regions with closed time-like curves may be excised as suggested in [45]. Moreover, the singularity in the cosmological regions may be resolved by the the same effects [12] possibly with the enhancement by string effects.

The effective action for the winding strings may be constructed from the correlation functions of twist fields. We have computed a general four point function of the form

$$
\left\langle V_{-k_{1}} V_{k_{2}} V_{-k_{3}} V_{k_{4}}\right\rangle
$$

which is related to the quartic term of the winding string interaction. Our result (4.11) implies that this quantity is finite, which may lead the following implications. Consider the $2 \rightarrow 2$ scattering of winding strings. The finiteness of the scattering means that the winding string does not feel the singular property of the background contrary to strings in the untwisted sector. Moreover, we may be able to apply to the tachyon condensation. In Euclidean orbifold case, it was shown in [46] that the condensation of localized tachyon changes the geometry, say, from $\mathbb{C} / \mathbb{Z}_{N}$ into $\mathbb{C} / \mathbb{Z}_{M}$ with $M<N$. The height of tachyon potential is proposed to be the same as the difference of the geometry volume [47], and 
this proposal was investigated by means of string field theory $[48,49]$ and effective field theory $[50,51]$. Since we found the quartic term is finite, it has a meaning to study whether a similar situation would happen or not.

We would like to investigate the following problems. An interesting problem is whether the tachyon condensation would separate the big crunch region and the big bang region. In order to answer this question, we may need to develop a way to sum over all contribution in the abbreviation of (5.2). We could expect that this investigation gives insights to the property of the tachyon state $[12,13]$. It is also worth applying our method to more general backgrounds. In particular, the parabolic or null orbifold case [4, 5] seems interesting because of the presence of supersymmetry. We hope that we could report on this subject in near future. The holographic dual description is also important because it might give a non-perturbative picture of the resolution of the singularity as mentioned before.

\section{Acknowledgement}

We would like to thank N. Iizuka, I. Papadimitriou, S. Ribault, V. Schomerus and J. Teschner for useful discussions. This work is supported by JSPS Postdoctoral Fellowships for Research Abroad H18-143.

\section{A Formula for hypergeometric function}

The hypergeometric function is defined as

$$
F(\alpha, \beta, \gamma ; z)=\sum_{n=0}^{\infty} \frac{(\alpha)_{n}(\beta)_{n}}{(\gamma)_{n}} \frac{z^{n}}{n !}, \quad(\alpha)_{n}=\frac{\Gamma(\alpha+n)}{\Gamma(\alpha)},
$$

and the integral expression is given by

$$
F(\alpha, \beta, \gamma ; z)=\frac{\Gamma(\gamma)}{\Gamma(\beta) \Gamma(\gamma-\beta)} \int_{0}^{1} d t t^{\beta-1}(1-t)^{\gamma-\beta-1}(1-t z)^{-\alpha}
$$

We use the following relation involving the derivative

$$
\begin{aligned}
& \gamma(1-z) \partial_{z} F(\alpha, \beta, \gamma ; z) \\
& \quad=(\gamma-\alpha)(\gamma-\beta) F(\alpha, \beta, \gamma+1 ; z)+\gamma(\alpha+\beta-\gamma) F(\alpha, \beta, \gamma ; z) .
\end{aligned}
$$

In order to see the asymptotic behavior of hypergeometric function, the following relations are useful as

$$
\begin{aligned}
F(\alpha, \beta, \gamma ; z) & =\frac{\Gamma(\gamma) \Gamma(\alpha+\beta-\gamma)}{\Gamma(\alpha) \Gamma(\beta)}(1-z)^{\gamma-\alpha-\beta} F(\gamma-\alpha, \gamma-\beta, \gamma-\alpha-\beta+1 ; 1-z) \\
& +\frac{\Gamma(\gamma) \Gamma(\gamma-\alpha-\beta)}{\Gamma(\gamma-\alpha) \Gamma(\gamma-\beta)} F(\alpha, \beta, \alpha+\beta-\gamma+1 ; 1-z)
\end{aligned}
$$




$$
\begin{aligned}
F(\alpha, \beta, \gamma ; z) & =\frac{\Gamma(\gamma) \Gamma(\beta-\alpha)}{\Gamma(\beta) \Gamma(\gamma-\alpha)}(-z)^{-\alpha} F\left(\alpha, \alpha-\gamma+1, \alpha-\beta+1 ; \frac{1}{z}\right) \\
& +\frac{\Gamma(\gamma) \Gamma(\alpha-\beta)}{\Gamma(\alpha) \Gamma(\gamma-\beta)}(-z)^{-\beta} F\left(\beta, \beta-\gamma+1, \beta-\alpha+1 ; \frac{1}{z}\right) \\
F(\alpha, \beta, \gamma ; z)= & \frac{\Gamma(\gamma) \Gamma(\beta-\alpha)}{\Gamma(\beta) \Gamma(\gamma-\alpha)}(1-z)^{-\alpha} F\left(\alpha, \gamma-\beta, \alpha-\beta+1 ; \frac{1}{1-z}\right) \\
& +\frac{\Gamma(\gamma) \Gamma(\alpha-\beta)}{\Gamma(\alpha) \Gamma(\gamma-\beta)}(1-z)^{-\beta} F\left(\beta, \gamma-\alpha, \beta-\alpha+1 ; \frac{1}{1-z}\right)
\end{aligned}
$$

Let us apply these relations to the functions defined in (4.23). For $x, \bar{x} \sim 0$, it is convenient to rewrite as

$$
\begin{aligned}
F_{2}(1-x)= & \frac{\Gamma\left(1+\frac{k_{2}}{N}-\frac{k_{3}}{N}\right) \Gamma\left(\frac{k_{1}}{N}-\frac{k_{2}}{N}\right)}{\Gamma\left(\frac{k_{1}}{N}\right) \Gamma\left(1-\frac{k_{3}}{N}\right)} x^{\frac{k_{2}}{N}-\frac{k_{1}}{N}} F\left(1-\frac{k_{4}}{N}, \frac{k_{2}}{N}, 1+\frac{k_{2}}{N}-\frac{k_{1}}{N} ; x\right) \\
+ & \frac{\Gamma\left(1+\frac{k_{2}}{N}-\frac{k_{3}}{N}\right) \Gamma\left(\frac{k_{2}}{N}-\frac{k_{1}}{N}\right)}{\Gamma\left(1-\frac{k_{4}}{N}\right) \Gamma\left(\frac{k_{2}}{N}\right)} F\left(\frac{k_{1}}{N}, 1-\frac{k_{3}}{N}, 1+\frac{k_{1}}{N}-\frac{k_{2}}{N} ; x\right), \\
\bar{G}_{2}(1-\bar{x})= & \frac{\Gamma\left(1+\frac{k_{3}}{N}-\frac{k_{2}}{N}\right) \Gamma\left(\frac{k_{2}}{N}-\frac{k_{1}}{N}\right)}{\Gamma\left(\frac{k_{3}}{N}\right) \Gamma\left(1-\frac{k_{1}}{N}\right)} \bar{x}^{\frac{k_{1}}{N}-\frac{k_{2}}{N}} F\left(\frac{k_{4}}{N}, 1-\frac{k_{2}}{N}, 1+\frac{k_{1}}{N}-\frac{k_{2}}{N} ; \bar{x}\right) \\
+ & \frac{\Gamma\left(1+\frac{k_{3}}{N}-\frac{k_{2}}{N}\right) \Gamma\left(\frac{k_{1}}{N}-\frac{k_{2}}{N}\right)}{\Gamma\left(\frac{k_{4}}{N}\right) \Gamma\left(1-\frac{k_{2}}{N}\right)} F\left(1-\frac{k_{1}}{N}, \frac{k_{3}}{N}, 1+\frac{k_{2}}{N}-\frac{k_{1}}{N} ; \bar{x}\right),
\end{aligned}
$$

and for $x, \bar{x} \sim \infty$

$$
\begin{aligned}
F_{1}(x) & =\frac{\Gamma\left(1+\frac{k_{2}}{N}-\frac{k_{1}}{N}\right) \Gamma\left(1-\frac{k_{1}}{N}-\frac{k_{3}}{N}\right)}{\Gamma\left(1-\frac{k_{1}}{N}\right) \Gamma\left(1-\frac{k_{4}}{N}\right)}(-x)^{-\frac{k_{3}}{N}} F\left(\frac{k_{3}}{N}, \frac{k_{4}}{N}, \frac{k_{1}}{N}+\frac{k_{3}}{N} ; \frac{1}{x}\right) \\
& +\frac{\Gamma\left(1+\frac{k_{2}}{N}-\frac{k_{1}}{N}\right) \Gamma\left(-1+\frac{k_{1}}{N}+\frac{k_{3}}{N}\right)}{\Gamma\left(\frac{k_{3}}{N}\right) \Gamma\left(\frac{k_{2}}{N}\right)}(-x)^{-1+\frac{k_{1}}{N}} F\left(1-\frac{k_{1}}{N}, 1-\frac{k_{2}}{N}, 2-\frac{k_{1}}{N}-\frac{k_{3}}{N} ; \frac{1}{x}\right) \\
\bar{G}_{1}(\bar{x}) & =\frac{\Gamma\left(1+\frac{k_{1}}{N}-\frac{k_{2}}{N}\right) \Gamma\left(-1+\frac{k_{1}}{N}+\frac{k_{3}}{N}\right)}{\Gamma\left(\frac{k_{1}}{N}\right) \Gamma\left(\frac{k_{4}}{N}\right)}(-\bar{x})^{-1+\frac{k_{3}}{N}} F\left(1-\frac{k_{3}}{N}, 1-\frac{k_{4}}{N}, 2-\frac{k_{1}}{N}-\frac{k_{2}}{N} ; \frac{1}{\bar{x}}\right) \\
& +\frac{\Gamma\left(1+\frac{k_{1}}{N}-\frac{k_{2}}{N}\right) \Gamma\left(1-\frac{k_{1}}{N}-\frac{k_{3}}{N}\right)}{\Gamma\left(1-\frac{k_{3}}{N}\right) \Gamma\left(1-\frac{k_{2}}{N}\right)}(-\bar{x})^{-\frac{k_{1}}{N}} F\left(\frac{k_{1}}{N}, \frac{k_{2}}{N}, \frac{k_{1}}{N}+\frac{k_{3}}{N} ; \frac{1}{\bar{x}}\right)
\end{aligned}
$$

and

$$
\begin{aligned}
F_{2}(1-x) & =\frac{\Gamma\left(1+\frac{k_{2}}{N}-\frac{k_{3}}{N}\right) \Gamma\left(1-\frac{k_{1}}{N}-\frac{k_{3}}{N}\right)}{\Gamma\left(1-\frac{k_{3}}{N}\right) \Gamma\left(1-\frac{k_{4}}{N}\right)} x^{-\frac{k_{1}}{N}} F\left(\frac{k_{1}}{N}, \frac{k_{2}}{N}, \frac{k_{1}}{N}+\frac{k_{3}}{N} ; \frac{1}{x}\right) \\
& +\frac{\Gamma\left(1+\frac{k_{2}}{N}-\frac{k_{3}}{N}\right) \Gamma\left(-1+\frac{k_{1}}{N}+\frac{k_{3}}{N}\right)}{\Gamma\left(\frac{k_{1}}{N}\right) \Gamma\left(\frac{k_{2}}{N}\right)} x^{-1+\frac{k_{3}}{N}} F\left(1-\frac{k_{3}}{N}, 1-\frac{k_{4}}{N}, 2-\frac{k_{1}}{N}-\frac{k_{3}}{N} ; \frac{1}{x}\right)
\end{aligned}
$$




$$
\begin{aligned}
\bar{G}_{2}(1-\bar{x}) & =\frac{\Gamma\left(1+\frac{k_{3}}{N}-\frac{k_{2}}{N}\right) \Gamma\left(-1+\frac{k_{1}}{N}+\frac{k_{3}}{N}\right)}{\Gamma\left(\frac{k_{3}}{N}\right) \Gamma\left(\frac{k_{4}}{N}\right)} \bar{x}^{-1+\frac{k_{1}}{N}} F\left(1-\frac{k_{1}}{N}, 1-\frac{k_{2}}{N}, 2-\frac{k_{1}}{N}-\frac{k_{3}}{N} ; \frac{1}{\bar{x}}\right) \\
& +\frac{\Gamma\left(1+\frac{k_{3}}{N}-\frac{k_{2}}{N}\right) \Gamma\left(1-\frac{k_{1}}{N}-\frac{k_{3}}{N}\right)}{\Gamma\left(1-\frac{k_{1}}{N}\right) \Gamma\left(1-\frac{k_{2}}{N}\right)} \bar{x}^{-\frac{k_{3}}{N}} F\left(\frac{k_{3}}{N}, \frac{k_{4}}{N}, \frac{k_{1}}{N}+\frac{k_{3}}{N} ; \frac{1}{\bar{x}}\right)
\end{aligned}
$$

\section{References}

[1] L. Cornalba and M. S. Costa, "Time-dependent orbifolds and string cosmology," Fortsch. Phys. 52 (2004) 145-199, hep-th/0310099.

[2] B. Durin and B. Pioline, "Closed strings in Misner space: A toy model for a big bounce?," hep-th/0501145.

[3] B. Craps, "Big bang models in string theory," hep-th/0605199.

[4] H. Liu, G. W. Moore, and N. Seiberg, "Strings in a time-dependent orbifold," JHEP 06 (2002) 045, hep-th/0204168.

[5] H. Liu, G. W. Moore, and N. Seiberg, "Strings in time-dependent orbifolds," JHEP 10 (2002) 031, hep-th/0206182.

[6] M. Berkooz, B. Craps, D. Kutasov, and G. Rajesh, "Comments on cosmological singularities in string theory," JHEP 03 (2003) 031, hep-th/0212215.

[7] G. T. Horowitz and J. Polchinski, "Instability of spacelike and null orbifold singularities," Phys. Rev. D66 (2002) 103512, hep-th/0206228.

[8] J. Figueroa-O'Farrill and J. Simon, "Generalized supersymmetric fluxbranes," JHEP 12 (2001) 011, hep-th/0110170.

[9] M. Fabinger and J. McGreevy, "On smooth time-dependent orbifolds and null singularities," JHEP 06 (2003) 042, hep-th/0206196.

[10] M. Berkooz, B. Pioline, and M. Rozali, "Closed strings in Misner space," JCAP 0408 (2004) 004, hep-th/0405126.

[11] M. Berkooz, B. Durin, B. Pioline, and D. Reichmann, "Closed strings in Misner space: Stringy fuzziness with a twist," JCAP 0410 (2004) 002, hep-th/0407216.

[12] J. McGreevy and E. Silverstein, "The tachyon at the end of the universe," JHEP 08 (2005) 090, hep-th/0506130.

[13] Y. Nakayama, S.-J. Rey, and Y. Sugawara, "The nothing at the beginning of the universe made precise," hep-th/0606127.

[14] M. Berkooz, Z. Komargodski, D. Reichmann, and V. Shpitalnik, "Flow of geometries and instantons on the null orbifold," JHEP 12 (2005) 018, hep-th/0507067.

[15] J.-H. She, "A matrix model for Misner universe," JHEP 01 (2006) 002, hep-th/0509067.

[16] Y. Hikida and T.-S. Tai, "D-instantons and closed string tachyons in Misner space," JHEP 01 (2006) 054, hep-th/0510129. 
[17] B. Craps, S. Sethi, and E. P. Verlinde, "A matrix big bang," JHEP 10 (2005) 005, hep-th/0506180.

[18] D. Robbins and S. Sethi, "A matrix model for the null-brane," JHEP 02 (2006) 052, hep-th/0509204.

[19] E. J. Martinec, D. Robbins, and S. Sethi, "Toward the end of time," hep-th/0603104.

[20] M. Li, "A class of cosmological matrix models," Phys. Lett. B626 (2005) 202-208, hep-th/0506260.

[21] M. Li and W. Song, "Shock waves and cosmological matrix models," JHEP 10 (2005) 073, hep-th/0507185.

[22] S. R. Das and J. Michelson, "pp wave big bangs: Matrix strings and shrinking fuzzy spheres," Phys. Rev. D72 (2005) 086005, hep-th/0508068.

[23] B. Chen, "The time-dependent supersymmetric configurations in M-theory and matrix models," Phys. Lett. B632 (2006) 393-398, hep-th/0508191.

[24] T. Ishino, H. Kodama, and N. Ohta, "Time-dependent solutions with null Killing spinor in M-theory and superstrings," Phys. Lett. B631 (2005) 68-73, hep-th/0509173.

[25] M. Li and W. Song, "A one loop problem of the matrix big bang model," hep-th/0512335.

[26] B. Craps, A. Rajaraman, and S. Sethi, "Effective dynamics of the matrix big bang," Phys. Rev. D73 (2006) 106005, hep-th/0601062.

[27] C.-S. Chu and P.-M. Ho, "Time-dependent AdS/CFT duality and null singularity," JHEP 04 (2006) 013, hep-th/0602054.

[28] S. R. Das and J. Michelson, "Matrix membrane big bangs and D-brane production," hep-th/0602099.

[29] S. R. Das, J. Michelson, K. Narayan, and S. P. Trivedi, "Time dependent cosmologies and their duals," hep-th/0602107.

[30] F.-L. Lin and W.-Y. Wen, "Supersymmteric null-like holographic cosmologies," hep-th/0602124.

[31] H.-Z. Chen and B. Chen, "Matrix model in a class of time dependent supersymmetric backgrounds," hep-th/0603147.

[32] T. Ishino and N. Ohta, "Matrix string description of cosmic singularities in a class of time-dependent solutions," hep-th/0603215.

[33] R. R. Nayak and K. L. Panigrahi, "D-brane solutions in a light-like linear dilaton background," hep-th/0604172.

[34] H. Kodama and N. Ohta, "Time-dependent supersymmetric solutions in M theory and compactification-decompactification transition," hep-th/0605179.

[35] R. R. Nayak, K. L. Panigrahi, and S. Siwach, "Time-dependent supergravity solutions in null dilaton background," hep-th/0605278. 
[36] G. D'Appollonio and E. Kiritsis, "String interactions in gravitational wave backgrounds," Nucl. Phys. B674 (2003) 80-170, hep-th/0305081.

[37] Y.-K. E. Cheung, L. Freidel, and K. Savvidy, "Strings in gravimagnetic fields," JHEP 02 (2004) 054, hep-th/0309005.

[38] M. Bianchi, G. D'Appollonio, E. Kiritsis, and O. Zapata, "String amplitudes in the Hpp-wave limit of $A d S_{3} \times S^{3}, " J H E P 04$ (2004) 074, hep-th/0402004.

[39] N. A. Nekrasov, "Milne universe, tachyons, and quantum group," Surveys High Energ. Phys. 17 (2002) 115-124, hep-th/0203112.

[40] B. Pioline and M. Berkooz, "Strings in an electric field, and the Milne universe," JCAP 0311 (2003) 007, hep-th/0307280.

[41] Y. Hikida, R. R. Nayak, and K. L. Panigrahi, "D-branes in a big bang/big crunch universe: Misner space," JHEP 09 (2005) 023, hep-th/0508003.

[42] T.-S. Tai, "D-branes in Lorentzian Melvin geometry," hep-th/0601039.

[43] L. J. Dixon, D. Friedan, E. J. Martinec, and S. H. Shenker, "The conformal field theory of orbifolds," Nucl. Phys. B282 (1987) 13-73.

[44] S. Hamidi and C. Vafa, "Interactions on orbifolds," Nucl. Phys. B279 (1987) 465.

[45] M. S. Costa, C. A. R. Herdeiro, J. Penedones, and N. Sousa, "Hagedorn transition and chronology protection in string theory," Nucl. Phys. B728 (2005) 148-178, hep-th/0504102.

[46] A. Adams, J. Polchinski, and E. Silverstein, "Don't panic! Closed string tachyons in ALE space-times," JHEP 10 (2001) 029, hep-th/0108075.

[47] A. Dabholkar, "Tachyon condensation and black hole entropy," Phys. Rev. Lett. 88 (2002) 091301, hep-th/0111004.

[48] Y. Okawa and B. Zwiebach, "Twisted tachyon condensation in closed string field theory," JHEP 03 (2004) 056, hep-th/0403051.

[49] O. Bergman and S. S. Razamat, "On the CSFT approach to localized closed string tachyons," JHEP 01 (2005) 014, hep-th/0410046.

[50] A. Dabholkar, A. Iqubal, and J. Raeymaekers, "Off-shell interactions for closed-string tachyons," JHEP 05 (2004) 051, hep-th/0403238.

[51] M. Headrick and J. Raeymaekers, "The large N limit of $\mathbb{C} / \mathbb{Z}_{N}$ and supergravity," JHEP 02 (2005) 054, hep-th/0411148. 